\title{
TWO NEW WEAK CONSTRAINT QUALIFICATIONS AND APPLICATIONS*
}

\author{
ROBERTO ANDREANI ${ }^{\dagger}$, GABRIEL HAESER ${ }^{\ddagger}$, MARÍA LAURA SCHUVERDT $^{\S}$, AND \\ PAULO J. S. SILVA
}

\begin{abstract}
We present two new constraint qualifications (CQs) that are weaker than the recently introduced relaxed constant positive linear dependence (RCPLD) CQ. RCPLD is based on the assumption that many subsets of the gradients of the active constraints preserve positive linear dependence locally. A major open question was to identify the exact set of gradients whose properties had to be preserved locally and that would still work as a CQ. This is done in the first new CQ, which we call the constant rank of the subspace component (CRSC) CQ. This new CQ also preserves many of the good properties of RCPLD, such as local stability and the validity of an error bound. We also introduce an even weaker CQ, called the constant positive generator (CPG), which can replace RCPLD in the analysis of the global convergence of algorithms. We close this work by extending convergence results of algorithms belonging to all the main classes of nonlinear optimization methods: sequential quadratic programming, augmented Lagrangians, interior point algorithms, and inexact restoration.
\end{abstract}

Key words. constraint qualifications, error bound, algorithmic convergence

AMS subject classifications. 90C46, 90C30

DOI. $10.1137 / 110843939$

1. Introduction. Let us consider a nonlinear optimization problem in the form

$$
\begin{array}{cl}
\min & f_{0}(x) \\
\text { s.t. } & f_{i}(x)=0, i=1, \ldots, m, \\
& f_{j}(x) \leq 0, j=m+1, \ldots, m+p,
\end{array}
$$

where the functions $f_{i}: \mathbb{R}^{n} \rightarrow \mathbb{R}, i=0, \ldots, m+p$, are continuously differentiable. We denote its feasible set by $F$. The constraints that hold as equalities in a point $x$ are said to be active at $x$. If $x$ is a feasible point, then the active constraints contain all the equality constraints together with a possibly empty subset of inequalities. We will denote by $\mathcal{A}(x)$ the index set of the active inequality constraints $\mathcal{A}(x) \stackrel{\text { def }}{=}\{i \mid$ $\left.f_{i}(x)=0, i=m+1, \ldots, m+p\right\}$.

One of the main subjects in the theory of nonlinear optimization is the characterization of optimality, which is often achieved through conditions that use the derivatives of the constraints at a prospective optimum. Among such conditions,

*Received by the editors August 9, 2011; accepted for publication (in revised form) May 29, 2012; published electronically September 13, 2012. This work was supported by PRONEXOptimization (PRONEX-CNPq/FAPERJ E-26/171.510/2006-APQ1), Fapesp (grants 2006/53768-0, 2009/09414-7, and 2010/19720-5), and CNPq (grants 300900/2009-0, 303030/2007-0, 305740/2010-5, and 474138/2008-9).

http://www.siam.org/journals/siopt/22-3/84393.html

$\dagger$ Department of Applied Mathematics, Institute of Mathematics, Statistics and Scientific Computing, University of Campinas, Campinas, SP, Brazil (andreani@ime.unicamp.br).

${ }^{\ddagger}$ Institute of Science and Technology, Federal University of São Paulo, São José dos Campos, SP, Brazil (gabriel.haeser@unifesp.br).

$\S$ CONICET, Department of Mathematics, FCE, University of La Plata, CP 172, 1900 La Plata Bs. As., Argentina (schuverd@mate.unlp.edu.ar).

IInstitute of Mathematics and Statistics, University of São Paulo, São Paulo, SP, Brazil (pjssilva@ ime.usp.br). 
arguably the most important is the Karush-Kuhn-Tucker (KKT) condition, which is extensively used in the development of algorithms to solve (NOP) [8,35].

In order to ensure that the KKT conditions are necessary for optimality a constraint qualification (CQ) is needed. CQs are properties of the algebraic description of the feasible set that allow its local geometry at a feasible point $x$ to be recovered from the gradients of the active constraints at $x$. In order to make this sentence clear we need to recall some definitions.

Definition 1.1. Let $x$ be a feasible point of (NOP), that is, $x \in F$. The tangent cone of $F$ at $x$ is defined as

$$
\mathcal{T}(x) \stackrel{\text { def }}{=}\left\{\begin{array}{l|l}
y \in \mathbb{R}^{n} & \begin{array}{c}
\exists x^{k} \in F, x^{k} \rightarrow x \\
\frac{x^{k}-x}{\left\|x^{k}-x\right\|} \rightarrow \frac{y}{\|y\|}
\end{array}
\end{array}\right\} \cup\{0\} .
$$

This cone is composed by the limit of directions that move inward of the feasible set. It is inherently a geometric object, as it captures the local "shape" of the set around $x$. Using it, we can easily present a geometric necessary optimality condition for local optimality at $x$ :

$$
-\nabla f_{0}(x) \in \mathcal{T}(x)^{\circ}
$$

where $\mathcal{T}(x)^{\circ}$ is the polar of $\mathcal{T}(x)[8]$.

However, the tangent cone is not an algebraic object, and hence it cannot be directly used in algorithms. CQs are conditions that ensure that $\mathcal{T}(x)^{\circ}$ can be recast using the algebraic information of the gradients. More specifically, we may try to approximate the tangent cone using the linearized cone of $F$ at $x$, which uses only information of the gradients and is given by

$$
\mathcal{F}(x) \stackrel{\text { def }}{=}\left\{y \mid \nabla f_{i}(x)^{\prime} y=0, i \in 1, \ldots, m, \nabla f_{j}(x)^{\prime} y \leq 0, j \in \mathcal{A}(x)\right\} .
$$

Note that this cone always contains the tangent cone; that is, $\mathcal{T}(x) \subset \mathcal{F}(x)$.

The polar of $\mathcal{F}(x)$ can be computed easily and is given by

$$
\mathcal{F}(x)^{\circ}=\left\{y \mid y=\sum_{i=1}^{m} \lambda_{i} \nabla f_{i}(x)+\sum_{j \in \mathcal{A}(x)} \mu_{j} \nabla f_{j}(x), \mu_{j} \geq 0\right\} .
$$

If $\mathcal{F}(x)^{\circ}=\mathcal{T}(x)^{\circ}$, then the optimality condition (1.1) can be rewritten as

$$
-\nabla f_{0}(x) \in \mathcal{F}(x)^{\circ}
$$

which is exactly the KKT condition. The condition $\mathcal{F}(x)^{\circ}=\mathcal{T}(x)^{\circ}$ was introduced by Guignard [15], and the discussion above suggests that it is the most general CQ possible. In fact, Gould and Tolle proved in [13] that it is equivalent to the necessity of the KKT condition for all possible objective functions.

Another possibility is to require directly that $\mathcal{F}(x)=\mathcal{T}(x)$. Even though this condition is more stringent than Guignard's CQ, it is in some cases easier to work with since it does not involve the polar operation. Such a CQ was introduced by Abadie in [1], and it is widely used in optimization theory [41, 7, 9, 26].

Clearly both Guignard's and Abadie's CQs are enforcing the equality between geometric objects that capture the local structure of the feasible set around $x$, namely, 
$\mathcal{T}(x)$ and its polar, with objects that use gradient information at the point $x$. The gradients have local information of the respective constraint functions, but they cannot always express the interrelationship among all functions while defining the feasible set. In this sense, we can say that a CQ is a condition that tries to restrict how the gradients, and hence the constraints themselves, vary together in a neighborhood of $x$. Such variation should be well behaved enough to assert Guignard's condition.

The simplest CQ, called the linear independence $C Q$ (LICQ), asks for linear independence of the gradients of the active constraints at the point of interest $x$. This condition is still important today and is required in many special cases, especially when connected to convergence results for numerical algorithms [8, 35]. When the problem has inequality constraints it is usually better to consider the MangasarianFromovitz CQ (MFCQ), which asks that the gradients of the active constraints be positively linearly independent, ${ }^{1}$ relaxing the LICQ $[27,40]$. Even though these two conditions appear to be pointwise conditions, they actually constrain how the gradients may vary together in a neighborhood of $x$, as linear independence and positive linear independence are conditions that are preserved locally.

The LICQ was relaxed by Janin in [22] while studying the directional derivative of the marginal function associated with the right-hand side of (NOP). In particular, Janin showed that if the ranks of all subsets of the gradients of the active constraints remain constant in a neighborhood of $x$, then the KKT conditions are necessary for optimality. This condition is known as the constant rank CQ (CRCQ). Clearly, LICQ is a particular case of CRCQ.

The CRCQ was further relaxed by Qi and Wei [38] in the context of studying sequential quadratic programming algorithms. The authors introduced the constant positive linear dependence (CPLD) condition, which was shown to be a CQ by Andreani, Martínez, and Schuverdt [6]. In [2,3] Andreani et al. showed that this CQ was enough to ensure the convergence of an augmented Lagrangian method to a KKT point. The CPLD condition asks that the positive linear dependence of any subset of the active gradients be preserved locally.

More recently, Minchenko and Stakhovski showed that the CRCQ can be relaxed to consider only the full set of the equality constraints [32]. More precisely, they showed that the following condition is a CQ.

DEFINITION 1.2. We say that the relaxed constant rank $C Q$ (RCRCQ) holds at a feasible point $x$ if there is a neighborhood $N(x)$ of $x$, where for all subsets $\mathcal{J} \subset \mathcal{A}(x)$ and all $y \in N(x)$ the set of gradients $\left\{\nabla f_{i}(y) \mid i \in\{1, \ldots, m\} \cup \mathcal{J}\right\}$ has constant rank.

Interesting relations between this condition and the original constant rank condition were unveiled in [24]. The relaxed constant rank condition was further extended to take into account positive linear independence in the place of the rank in [5], where a relaxed version of the CPLD, called RCPLD, is introduced. This work also shows that RCPLD is enough to ensure the validity of an error bound and the global convergence of an augmented Lagrangian method.

These last developments are interesting as they do not take into account all the subsets of the gradients of the equality constraints. Only the full set of gradients $\left\{\nabla f_{1}(x), \ldots, \nabla f_{m}(x)\right\}$ is important. So, if the problem has only equality constraints, these conditions basically require that the linearized cone of $F$ must have constant dimension locally, only tilting to support the feasible set at each point. This is a strong geometric condition that is easy to understand and visualize.

\footnotetext{
${ }^{1}$ For a precise definition of positive linear independence in this context, see section 2.
} 


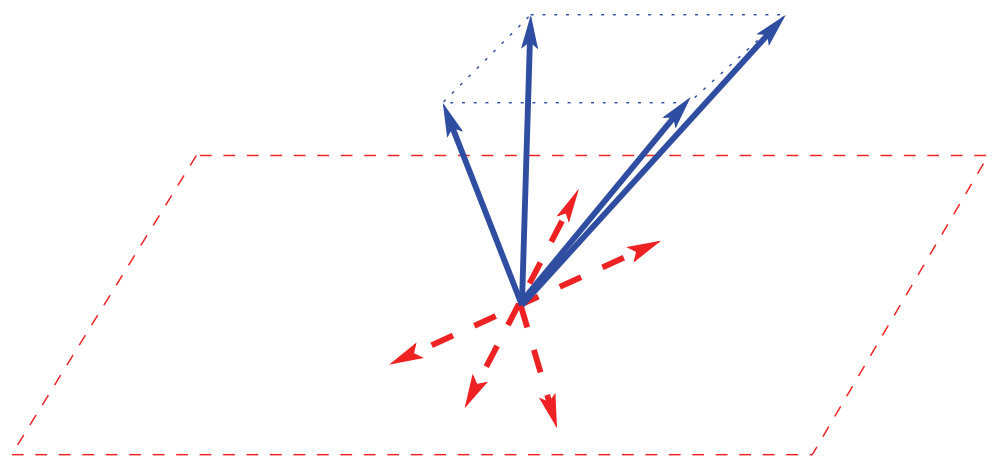

FIG. 1.1. Linear space and pointed cone components of $\mathcal{F}(x)^{\circ}$. The subspace is generated by the gradients of the equality constraints together with the gradients of constraints with indexes in $\mathcal{J}_{-}$. The pointed cone is generated by the gradients of the active inequality constraints that are not in $\mathcal{J}_{-}$.

However, if the problem has inequalities, the results described above still require local conditions on all subsets of the gradients of the active inequalities. The simplicity of considering only one set of gradients whose properties must be stable is lost. The main purpose of this paper is to fill this gap, showing that only a single subset of the inequality constraints needs to be considered.

When the feasible set is described with inequalities, the rank preservation of the gradients is not the right concept to describe its structure. For example, consider the constraints $y \geq 0, y-x^{2} \geq 0$. They conform to MFCQ at 0 , but their rank increases locally. The rank is a tool that is better suited to dealing with the gradients of the equality constraints as they generate a subspace contained in $\mathcal{F}(x)^{\circ}$ where the notion of dimension can be applied.

For inequality constraints the idea of CPLD looks like the best choice. On the other hand, in some cases, inequality constraints may behave like, or even be, equality constraints in disguise. For example, $x \geq 0$ and $x \leq 0$, which together mean $x=0$. In this case, rank preservation is the right concept.

How do we reconcile these two possibilities? One way is to try to identify which inequalities actually behave like equalities in the description of the polar of the linearized cone. With this objective in mind, let us consider the maximal subspace contained in $\mathcal{F}(x)^{\circ}$, which we call its subspace component. The description given in (1.3) seems to suggest that this subspace is generated by the gradients of the equalities. The other term in the sum, associated with the gradients of the inequalities, is expected to be a pointed cone. Most of the problems arise when this division is not clear, that is, when gradients of inequality constraints fall into the subspace component of the polar of the linearized cone. See Figure 1.1. Formally this happens whenever the set

$$
\mathcal{J}_{-} \stackrel{\text { def }}{=}\left\{j \in \mathcal{A}(x) \mid-\nabla f_{j}(x) \in \mathcal{F}(x)^{\circ}\right\}
$$

is nonempty. This index set appears implicitly in the MFCQ, which is equivalent to requiring that $\mathcal{J}_{-}$be empty, while the gradients of the equality constraints that generate the linear space component of the polar of the linearized cone must be linearly independent, thus preserving its dimension locally.

In order to generalize the CQs described above, we need to generalize the notion of a basis of a subspace to deal with cones spanned by linear combinations using signed 
coefficients. We then require that such special spanning sets be preserved locally. The precise definition of this new CQ is given in section 3. In particular, we show that many of the CQs discussed above imply that the subspace component of the polar of the linearized cone has the same dimension locally, which in turn implies the new CQ.

The preservation of the dimension of the subspace component is an intermediate $\mathrm{CQ}$ that plays a fundamental role in the applications. Let us formalize it below.

DeFINITION 1.3. Let $x$ be a feasible point of (NOP), and define the index set $\mathcal{J}_{-}$as in (1.4). We say that the constant rank of the subspace component (CRSC) condition holds at $x$ if there is a neighborhood $N(x)$ of $x$ such that the rank of $\left\{\nabla f_{l}(y) \mid\right.$ $\left.l \in\{1, \ldots, m\} \cup \mathcal{J}_{-}\right\}$remains constant for $y \in N(x)$.

Note that the fact that CPLD CQs, in particular RCPLD, imply CRSC as proved in Theorem 4.3 is somewhat surprising. In particular, this fact reconciles constant rank and CPLD CQs: both are actually ensuring that the subspace spanned by the gradients of the equality constraints and the gradients of the inequality constraints with indexes in $\mathcal{J}_{-}$has constant dimension locally. The fact that the dimension of the linear space component is locally constant has deep geometrical consequences: it basically says that the polar of the linearized cone has the same shape locally; it can only tilt preserving its structure. Moreover, this condition is clearly more general than RCPLD, as the simple feasible set $\left\{x \mid x \leq 0,-x \leq 0, x^{2} \leq 0\right\}$ conforms to CRSC at its only point, the origin, while RCPLD fails.

The rest of this paper is organized as follows. Section 2 introduces the notion of positively linearly independent spanning pairs, which replaces the idea of a basis for cones. Section 3 uses this idea to introduce a new $\mathrm{CQ}$ that we call the constant positive generator (CPG) condition and that generalizes CRSC and many of the CQs described above. Section 4 shows the relation among RCPLD, CRSC, and CPG. It shows that CPG implies Abadie's CQ. Finally, section 5 shows some important applications of CRSC and CPG. We discuss when an error bound holds and also show that many algorithms converge under the weak CPG condition.

2. Positively linearly independent spanning pairs. One of the main objects in the study of $\mathrm{CQ}$ is $\mathcal{F}(x)^{\circ}$, the polar of the linearized cone of the feasible set at a feasible point $x$; see (1.3). This cone is spanned by the gradients of the active constraints at $x$ with some sign conditions on the combination coefficients. This notion of spanning cones using vectors and coefficients with sign conditions is fundamental in our development. Let us formalize it in the next definition.

Definition 2.1. Let $V=\left(v_{1}, v_{2}, \ldots, v_{K}\right)$ be a tuple $e^{2}$ of vectors in $\mathbb{R}^{n}$, and let $\mathcal{I}, \mathcal{J} \subset\{1,2, \ldots, K\}$ be a pair of index sets. We call a positive combination of elements of $V$ associated with the (ordered) pair $(\mathcal{I}, \mathcal{J})$ a vector in the form

$$
\sum_{i \in \mathcal{I}} \lambda_{i} v_{i}+\sum_{j \in \mathcal{J}} \mu_{j} v_{j}, \quad \mu_{j} \geq 0, \forall j \in \mathcal{J} .
$$

The set of all such positive combinations is called the positive span of $V$ associated with $(\mathcal{I}, \mathcal{J})$, and it is denoted by $\operatorname{span}_{+}(\mathcal{I}, \mathcal{J} ; V)$. It is clearly a cone. If the tuple $V$ is clear from the context, we may omit it and use positive combinations of $(\mathcal{I}, \mathcal{J})$, positive span of $(\mathcal{I}, \mathcal{J})$, and write $\operatorname{span}_{+}(\mathcal{I}, \mathcal{J})$. On the other hand, if the set $\mathcal{I}=\emptyset$,

\footnotetext{
${ }^{2}$ We use a tuple instead of a regular set to allow for vectors to appear more than once. It is natural to consider this possibility in our discussion as the gradients of different constraints may be equal in a given point.
} 
that is if all coefficients are supposed to be nonnegative, we may talk about positive combinations of $V$ and positive span of $V$.

The vectors $v_{\ell}, \ell \in \mathcal{I} \cup \mathcal{J}$, or the pair $(\mathcal{I}, \mathcal{J})$ when $V$ is clear from the context, are said to be positively linearly independent if the only way to write the zero vector using positive combinations is to use trivial coefficients. Otherwise we say that the vectors, or the pair, are positively linearly dependent.

Let $\mathcal{I}^{\prime}, \mathcal{J}^{\prime} \subset\{1,2, \ldots, K\}$ be another pair of indexes. We say that $\left(\mathcal{I}^{\prime}, \mathcal{J}^{\prime}\right)$ positively spans $\operatorname{span}_{+}(\mathcal{I}, \mathcal{J} ; V)$ if $\operatorname{span}_{+}\left(\mathcal{I}^{\prime}, \mathcal{J}^{\prime} ; V\right)=\operatorname{span}_{+}(\mathcal{I}, \mathcal{J} ; V)$. We may also say that $\left(\mathcal{I}^{\prime}, \mathcal{J}^{\prime}\right)$ is a positive spanning pair for such a cone.

Now, let us recall the definition of the polar of the linearized cone $\mathcal{F}(x)^{\circ}$ given in (1.3). If we set $\mathcal{I}$ as the indexes of the equality constraints $\{1,2, \ldots, m\}, \mathcal{J}$ as the indexes of the inequality constraints that are active at $x$, that is $\mathcal{A}(x)$, and $V$ as the tuple of gradients with indexes in $\mathcal{I} \cup \mathcal{J}$, then $\mathcal{F}(x)^{\circ}$ is the positive span of $V$ associated with the pair $(\mathcal{I}, \mathcal{J})$.

Next, let us try to generalize the idea of a basis from linear spaces to positive spanned cones in the form $\operatorname{span}_{+}(\mathcal{I}, \mathcal{J} ; V)$. In other words, we want to define a "minimal" spanning pair for such a cone. A first attempt is to look for a positively linearly independent spanning pair for it; however, the usual technique for finding such a pair may not apply. For example, for $V=\left\{v_{1}=-1, v_{2}=1\right\} \subset \mathbb{R}, \mathcal{I}=\emptyset$, and $\mathcal{J}=\{1,2\}$, it is not possible to obtain such a pair simply by removing vectors from $\mathcal{I}$ and $\mathcal{J}$, as is possible in the linear case. In order to find such a spanning pair we need to remove vectors from $\mathcal{J}$ and put them into $\mathcal{I}$. In fact, $\mathcal{I}^{\prime}=\{1\}$ and $\mathcal{J}^{\prime}=\emptyset$ form a positively linearly independent spanning pair for the same cone. We make this procedure clear in the next result.

TheOrem 2.2. Let $V=\left(v_{1}, v_{2}, \ldots, v_{K}\right)$ be a tuple of vectors in $\mathbb{R}^{n}$ and $\mathcal{I}, \mathcal{J} \subset$ $\{1,2, \ldots, K\}$ such that the pair $(\mathcal{I}, \mathcal{J})$ is positively linearly dependent. Then the pair $\left(\mathcal{I}^{\prime}, \mathcal{J}^{\prime}\right)$ defined below positively spans $\operatorname{span}_{+}(\mathcal{I}, \mathcal{J} ; V)$.

1. If $\mathcal{I}$ is associated with linearly dependent vectors, define $\mathcal{I}^{\prime}$ as a proper subset of $\mathcal{I}$ such that $\operatorname{span}\left\{v_{i} \mid i \in \mathcal{I}^{\prime}\right\}=\operatorname{span}\left\{v_{i} \mid i \in \mathcal{I}\right\}$ and set $\mathcal{J}^{\prime}=\mathcal{J}$.

2. Otherwise, $\mathcal{I}$ is associated with linearly independent vectors, and there is a $j^{\prime} \in \mathcal{J}$ such that $-v_{j} \in \operatorname{span}_{+}(\mathcal{I}, \mathcal{J})$. Define $\mathcal{I}^{\prime}=\mathcal{I} \cup\left\{j^{\prime}\right\}$ and $\mathcal{J}^{\prime}=\mathcal{J} \backslash\left\{j^{\prime}\right\}$, a proper subset of $\mathcal{J}$.

Proof. In the first case it is trivial to see that the cones coincide.

In the second case, as $(\mathcal{I}, \mathcal{J})$ is positively linearly dependent, there must be coefficients $\bar{\lambda}_{i}$, for $i \in \mathcal{I}$, and nonnegative $\bar{\mu}_{j}$, for $j \in \mathcal{J}$, such that

$$
\sum_{i \in \mathcal{I}} \bar{\lambda}_{i} v_{i}+\sum_{j \in \mathcal{J}} \bar{\mu}_{j} v_{j}=0
$$

Note that not all $\bar{\mu}_{j}, j \in \mathcal{J}$, are zero; otherwise, $v_{i}, i \in \mathcal{I}$, would not be linearly independent. Then there is at least one $j^{\prime} \in \mathcal{J}$ such that $\bar{\mu}_{j^{\prime}}>0$. Dividing the equation above by $\bar{\mu}_{j^{\prime}}$, we get

$$
\sum_{i \in \mathcal{I}} \frac{\bar{\lambda}_{i}}{\bar{\mu}_{j^{\prime}}} v_{i}+\sum_{j \in \mathcal{J} \backslash\left\{j^{\prime}\right\}} \frac{\bar{\mu}_{j}}{\bar{\mu}_{j^{\prime}}} v_{j}=-v_{j^{\prime}} .
$$

Now define the index sets $\mathcal{I}^{\prime} \stackrel{\text { def }}{=} \mathcal{I} \cup\left\{j^{\prime}\right\}$ and $\mathcal{J}^{\prime} \stackrel{\text { def }}{=} \mathcal{J} \backslash\left\{j^{\prime}\right\}$. Clearly, $\operatorname{span}_{+}\left(\mathcal{I}^{\prime}, \mathcal{J}^{\prime}\right) \supset$ $\operatorname{span}_{+}(\mathcal{I}, \mathcal{J})$. On the other hand, let

$$
\sum_{i \in \mathcal{I}^{\prime}} \lambda_{i} v_{i}+\sum_{j \in \mathcal{J}^{\prime}} \mu_{j} v_{j}
$$


be an element of $\operatorname{span}_{+}\left(\mathcal{I}^{\prime}, \mathcal{J}^{\prime}\right)$. Then, it clearly belongs to $\operatorname{span}_{+}(\mathcal{I}, \mathcal{J})$ if the coefficient of $v_{j^{\prime}}$ is nonnegative. Otherwise,

$$
\begin{aligned}
\sum_{i \in \mathcal{I}^{\prime}} \lambda_{i} v_{i}+\sum_{j \in \mathcal{J}^{\prime}} \mu_{j} v_{j}= & \sum_{i \in \mathcal{I}} \lambda_{i} v_{i}+\lambda_{j^{\prime}} v_{j^{\prime}}+\sum_{j \in \mathcal{J}^{\prime}} \mu_{j} v_{j} \\
= & \sum_{i \in \mathcal{I}} \lambda_{i} v_{i}+\left|\lambda_{j^{\prime}}\right|\left(\sum_{i \in \mathcal{I}} \frac{\bar{\lambda}_{i}}{\bar{\mu}_{j^{\prime}}} v_{i}+\sum_{j \in \mathcal{J} \backslash\left\{j^{\prime}\right\}} \frac{\bar{\mu}_{j}}{\bar{\mu}_{j^{\prime}}} v_{j}\right) \\
& +\sum_{j \in \mathcal{J}^{\prime}} \mu_{j} v_{j},
\end{aligned}
$$

and we see that it is actually in $\operatorname{span}_{+}(\mathcal{I}, \mathcal{J})$.

We can then easily construct positively linearly independent spanning pairs.

Corollary 2.3. Let $V=\left(v_{1}, v_{2}, \ldots, v_{K}\right)$ be a tuple of vectors in $\mathbb{R}^{n}$, and let $\mathcal{I}, \mathcal{J} \subset\{1,2, \ldots, K\}$ be a pair of index sets. Then there exist $\mathcal{I}^{\prime}, \mathcal{J}^{\prime} \subset\{1,2, \ldots, K\}$ such that $\left(\mathcal{I}^{\prime}, \mathcal{J}^{\prime}\right)$ is positively linearly independent and $\operatorname{span}_{+}\left(\mathcal{I}^{\prime}, \mathcal{J}^{\prime} ; V\right)=\operatorname{span}(\mathcal{I}$, $\mathcal{J} ; V)$. We call such pairs positively linearly independent spanning pairs of $\operatorname{span}_{+}(\mathcal{I}, \mathcal{J} ; V)$.

Proof. Start with $(\mathcal{I}, \mathcal{J})$ and apply the construction given in Theorem 2.2 while possible. Clearly this can be done only a finite number of times, and the resulting pair $\left(\mathcal{I}^{\prime}, \mathcal{J}^{\prime}\right)$ is positively linearly independent.

The second case in Theorem 2.2 simply states that if both $v_{j}$ and $-v_{j}$ belong to $\operatorname{span}_{+}(\mathcal{I}, \mathcal{J})$ for some index $j \in \mathcal{J}$, then this index may have been misplaced and should be moved to $\mathcal{I}$. If we recall the natural definitions $\mathcal{I}, \mathcal{J}$, and $V$ when considering $\mathcal{F}(x)^{\circ}$, moving an index from $\mathcal{J}$ to $\mathcal{I}$ is associated with stating that an inequality constraint should be viewed as an equality, something which is not usual in optimization.

To see why this is acceptable, let us recall that $\mathcal{F}(x)^{\circ}$ is the polar to the linearized cone. The fact that an inequality constraint $f_{j}$ has both $\nabla f_{j}(x)$ and $-\nabla f_{j}(x)$ in $\mathcal{F}(x)^{\circ}$ implies that $\mathcal{F}(x)$, and hence $\mathcal{T}(x)$, lies in the subspace orthogonal to $\nabla f_{j}(x)$. That is, if we consider the feasible set $F, f_{j}$ is interacting with the other constraints that define it and behaving more closely like an equality constraint than like an inequality constraint.

We end this section with an alternative characterization of the positively linearly independent spanning pairs given above. We start with a definition, already suggested in the introduction.

Definition 2.4. Let $V=\left(v_{1}, v_{2}, \ldots, v_{K}\right)$ be a tuple of vectors in $\mathbb{R}^{n}$, and let $\mathcal{I}, \mathcal{J} \subset\{1,2, \ldots, K\}$ be a pair of index sets. Define

$$
\mathcal{J}_{-} \stackrel{\text { def }}{=}\left\{j \in \mathcal{J} \mid-v_{j} \in \operatorname{span}_{+}(\mathcal{I}, \mathcal{J} ; V)\right\} \quad \text { and } \quad \mathcal{J}_{+} \stackrel{\text { def }}{=} \mathcal{J} \backslash \mathcal{J}_{-}
$$

Lemma 2.5. Let $V=\left(v_{1}, v_{2}, \ldots, v_{K}\right)$ be a tuple of vectors in $\mathbb{R}^{n}$, and let $\mathcal{I}, \mathcal{J} \subset$ $\{1,2, \ldots, K\}$ be a pair of index sets. If $\left(\mathcal{I}^{\prime}, \mathcal{J}^{\prime}\right)$ is a positively linearly independent spanning pair for $\operatorname{span}_{+}(\mathcal{I}, \mathcal{J} ; V)$, then

1. $\mathcal{J}^{\prime} \subset \mathcal{J}_{+}$;

2. $\left(\mathcal{I}^{\prime}, \mathcal{J}_{+}\right)$is also a positively linearly independent spanning pair for $\operatorname{span}_{+}(\mathcal{I}, \mathcal{J} ; V)$;

3. $\mathcal{I}^{\prime} \subset \mathcal{I} \cup \mathcal{J}_{-}$, and it is composed of indexes of a basis of the subspace spanned by $\left\{v_{\ell} \mid \ell \in \mathcal{I} \cup \mathcal{J}_{-}\right\}$. 
Proof.

1. Let $\ell \in \mathcal{J}^{\prime}$. Suppose, by contradiction, that $\ell \notin \mathcal{J}_{+}$; in other words, $-v_{\ell} \in$ $\operatorname{span}_{+}(\mathcal{I}, \mathcal{J} ; V)=\operatorname{span}_{+}\left(\mathcal{I}^{\prime}, \mathcal{J}^{\prime} ; V\right)$. In this case,

$$
-v_{\ell}=\sum_{i \in \mathcal{I}^{\prime}} \lambda_{i} v_{i}+\sum_{j \in \mathcal{J}^{\prime} \backslash\{\ell\}} \mu_{j} v_{j}+\mu_{\ell} v_{\ell}, \quad \mu_{j} \geq 0 \forall j \in \mathcal{J}^{\prime},
$$

which implies

$$
0=\sum_{i \in \mathcal{I}^{\prime}} \lambda_{i} v_{i}+\sum_{j \in \mathcal{J}^{\prime} \backslash\{\ell\}} \mu_{j} v_{j}+\left(\mu_{\ell}+1\right) v_{\ell}, \quad \mu_{j} \geq 0 \forall j \in \mathcal{J}^{\prime}
$$

As $\left(\mu_{\ell}+1\right)>0$, this is a contradiction to the assumption that $\left(\mathcal{I}^{\prime}, \mathcal{J}^{\prime}\right)$ is positively linearly independent.

2. First, observe that as $\mathcal{J}^{\prime} \subset \mathcal{J}_{+}, \operatorname{span}_{+}(\mathcal{I}, \mathcal{J} ; V)=\operatorname{span}_{+}\left(\mathcal{I}^{\prime}, \mathcal{J}^{\prime} ; V\right) \subset$ $\operatorname{span}_{+}\left(\mathcal{I}^{\prime}, \mathcal{J}_{+} ; V\right) \subset \operatorname{span}_{+}(\mathcal{I}, \mathcal{J} ; V)$. Hence, $\left(\mathcal{I}^{\prime}, \mathcal{J}_{+}\right)$is also a spanning pair. Now, suppose in contradiction that it is positively linearly dependent; that is, there are coefficients $\lambda_{i}$ for $i \in \mathcal{I}^{\prime}$ and $\mu_{j} \geq 0$ for $j \in \mathcal{J}_{+}$, not all zero, such that

$$
\sum_{i \in \mathcal{I}^{\prime}} \lambda_{i} v_{i}+\sum_{j \in \mathcal{J}_{+}} \mu_{j} v_{j}=0
$$

Since $\left(\mathcal{I}^{\prime}, \mathcal{J}^{\prime}\right)$ is positively linearly independent, the vectors with indexes in $\mathcal{I}^{\prime}$ are linearly independent. Hence, at least one of the coefficients $\mu_{j^{\prime}}, j^{\prime} \in \mathcal{J}_{+}$, is strictly positive. We can then rearrange the above equality to solve for $-v_{j^{\prime}}$ and get a contradiction to the definition of $\mathcal{J}_{+}$.

3. If $j \in \mathcal{I}^{\prime}$, then $-v_{j} \in \operatorname{span}_{+}(\mathcal{I}, \mathcal{J} ; V)$. Hence, $j$ must belong to either $\mathcal{I}$ or $\mathcal{J}_{-}$by definition of such index sets. Now, clearly, the vectors with indexes in $\mathcal{I}^{\prime}$ are linearly independent, as $\left(\mathcal{I}^{\prime}, \mathcal{J}^{\prime}\right)$ is positively linearly independent. We need only show that any $v_{\ell}, \ell \in \mathcal{I} \cup \mathcal{J}_{-}$, is a linear combination of the vectors with indexes in $\mathcal{I}^{\prime}$. Now, as both $v_{\ell},-v_{\ell} \in \operatorname{span}_{+}\left(\mathcal{I}^{\prime}, \mathcal{J}^{\prime}\right)$, there must be coefficients $\lambda_{i}^{+}, \lambda_{i}^{-}, i \in \mathcal{I}^{\prime}$, and nonnegative $\mu_{j}^{+}, \mu_{j}^{-}, j \in \mathcal{J}^{\prime}$, such that

$$
\begin{aligned}
v_{\ell} & =\sum_{i \in \mathcal{I}^{\prime}} \lambda_{i}^{+} v_{i}+\sum_{j \in \mathcal{J}^{\prime}} \mu_{j}^{+} v_{j}, \\
-v_{\ell} & =\sum_{i \in \mathcal{I}^{\prime}} \lambda_{i}^{-} v_{i}+\sum_{j \in \mathcal{J}^{\prime}} \mu_{j}^{-} v_{j} .
\end{aligned}
$$

Summing up these two inequalities, we get

$$
0=\sum_{i \in \mathcal{I}^{\prime}}\left(\lambda_{i}^{+}+\lambda_{i}^{-}\right) v_{i}+\sum_{j \in \mathcal{J}^{\prime}}\left(\mu_{j}^{+}+\mu_{j}^{-}\right) v_{j}
$$

As $\left(\mathcal{I}^{\prime}, \mathcal{J}^{\prime}\right)$ is positively linearly independent, we know that all coefficients in the summation above are zero. Since for all $j \in \mathcal{J}^{\prime}, \mu_{j}^{+}, \mu_{j}^{-} \geq 0$ we conclude that for all $j \in \mathcal{J}^{\prime}, \mu_{j}^{+}=\mu_{j}^{-}=0$. It follows from (2.2) that $v_{\ell}$ is spanned by the vectors in $\mathcal{I}^{\prime}$. 
COROLlary 2.6. The positively linearly independent spanning pairs given by Corollary 2.3 have the form

$$
\mathcal{I}^{\prime} \subset \mathcal{I} \cup \mathcal{J}_{-}, \quad \mathcal{J}^{\prime}=\mathcal{J}_{+},
$$

where $\mathcal{I}^{\prime}$ is composed by indexes of a basis of the space spanned by $\left\{v_{\ell} \mid \ell \in \mathcal{I} \cup \mathcal{J}_{-}\right\}$.

Proof. This is an immediate consequence of the lemma above and the fact that the procedure described in Corollary 2.3 never moves vectors from $\mathcal{J}_{+}$to the set $\mathcal{I}^{\prime}$.

Corollary 2.7. The set $\operatorname{span}_{+}\left(\mathcal{I}, \mathcal{J}_{-}\right)$is a subspace.

Proof. By definition, $v_{j} \in \mathcal{J}_{-}$if and only if $-v_{j}$ is a positive linear combination of the other vectors in $\mathcal{I} \cup \mathcal{J}$. But in this positive combination the vectors in $\mathcal{J}_{+}$can appear only with zero coefficients; otherwise they would belong to $\mathcal{J}_{-}$.

3. Constant positive generators. Now we are ready to introduce a new CQ. DeFINITION 3.1. Consider the nonlinear optimization problem (NOP). For $y \in$ $\mathbb{R}^{n}$ define the tuple $G f(y) \stackrel{\text { def }}{=}\left(\nabla f_{1}(y), \nabla f_{2}(y), \ldots, \nabla f_{m+p}(y)\right)$. Let $x$ be a feasible point, and define the index sets $\mathcal{I} \stackrel{\text { def }}{=}\{1,2, \ldots, m\}$ and $\mathcal{J} \stackrel{\text { def }}{=} \mathcal{A}(x)$, the set of active inequality constraints.

We say that the CPG condition holds at $x$ if there is a positively linearly independent spanning pair $\left(\mathcal{I}^{\prime}, \mathcal{J}_{+}\right)$of $\operatorname{span}_{+}(\mathcal{I}, \mathcal{J} ; G f(x))$ such that

$$
\operatorname{span}_{+}\left(\mathcal{I}^{\prime}, \mathcal{J}_{+} ; G f(y)\right) \supset \operatorname{span}_{+}(\mathcal{I}, \mathcal{J} ; G f(y))
$$

for all $y$ in a neighborhood of $x$.

Note that we implicitly used Lemma 2.5 in this definition. Actually, if $\left(\mathcal{I}^{\prime}, \mathcal{J}^{\prime}\right)$ is a positively linearly independent spanning pair for $\operatorname{span}_{+}(\mathcal{I}, \mathcal{J} ; G f(x))$, the lemma says that $\left(\mathcal{I}^{\prime}, \mathcal{J}_{+}\right)$is also a spanning pair. As $\mathcal{J}_{+} \supset \mathcal{J}^{\prime}$, it may be easier to show that the inclusion (3.1) holds using $\mathcal{J}_{+}$in the place of a smaller $\mathcal{J}^{\prime}$. Hence, we decided to state the definition already using the larger index set $\mathcal{J}_{+}$.

Note also that if the inclusion required in CPG holds, then it must hold as an equality. This is not always true. For example, consider the feasible set

$$
F=\left\{\left(x_{1}, x_{2}\right) \in \mathbb{R}^{2} \mid x_{1}^{3}-x_{2} \leq 0, x_{1}^{3}+x_{2} \leq 0, x_{1} \leq 0\right\}
$$

at the origin. At this point CPG holds with the inclusion holding in the proper sense. See Figure 3.1.

Finally, an extension of this example can also be used to show that it is possible for inclusion (3.1) to hold only for a specific choice for $\mathcal{I}^{\prime}$. In order to see this, let us add a constraint to the feasible set above and consider

$$
F=\left\{\left(x_{1}, x_{2}\right) \in \mathbb{R}^{2} \mid x_{1}^{3}-x_{2} \leq 0, x_{1}^{3}+x_{2} \leq 0, x_{1} \leq 0, x_{2}^{3} \leq 0\right\}
$$

at the origin. Here, the constraints associated with $\mathcal{J}_{-}$are the first, second, and fourth; that is, $\mathcal{J}_{-}=\{1,2,4\}$, while $\mathcal{J}_{+}=\{3\}$ and $\mathcal{I}=\emptyset$. There are two possible choices for $\mathcal{I}^{\prime}$ that are associated with positively linearly independent spanning pairs at the origin. Either $\mathcal{I}^{\prime}=\{1\}$, which shows that CPG holds, or $\mathcal{I}^{\prime}=\{2\}$, where the inclusion in the CPG definition is not valid. See Figure 3.2.

Now we move to proving that CPG is actually a CQ. First let us recall the definition of approximate KKT points [4].

DeFINITION 3.2. We say that a feasible point $x$ of (NOP) conforms to the approximate KKT (AKKT) condition if there exist sequences $x^{k} \rightarrow x, \epsilon^{k} \rightarrow 0$, and 


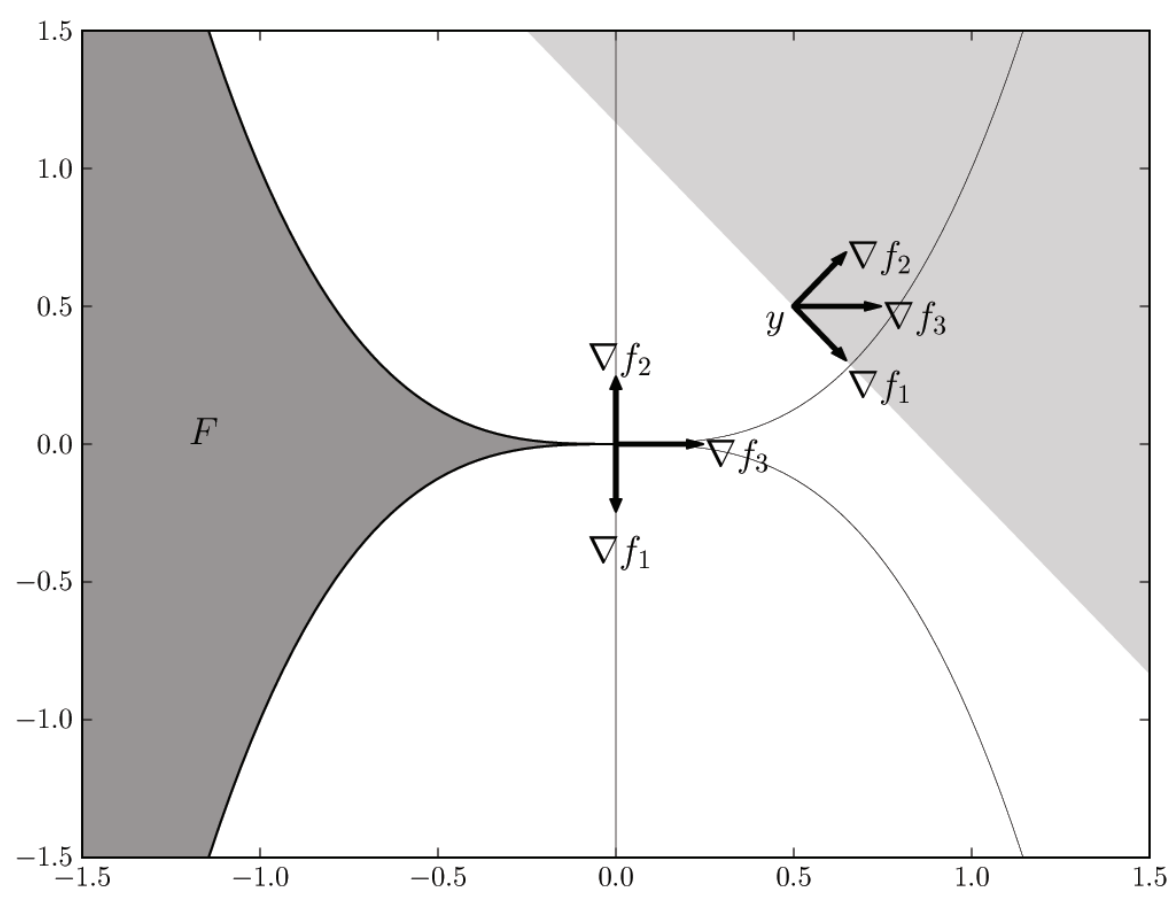

FIG. 3.1. Consider $F=\left\{\left(x_{1}, x_{2}\right) \in \mathbb{R}^{2} \mid f_{1}\left(x_{1}, x_{2}\right)=x_{1}^{3}-x_{2} \leq 0, f_{2}\left(x_{1}, x_{2}\right)=x_{1}^{3}+x_{2} \leq\right.$ $\left.0, f_{3}\left(x_{1}, x_{2}\right)=x_{1} \leq 0\right\}$ at the origin. Then we can take $\mathcal{I}^{\prime}=\{1\}$ and $\mathcal{J}_{+}=\{3\}$ in the definition of $C P G$. Then, for all $y \neq 0, \operatorname{span}_{+}(\{1\},\{3\} ; G f(y))$ is a semispace, pictured in light gray above, that properly contains the pointed cone $\operatorname{span}_{+}(\emptyset,\{1,2,3\} ; G f(y))$, positively generated by the gradients.

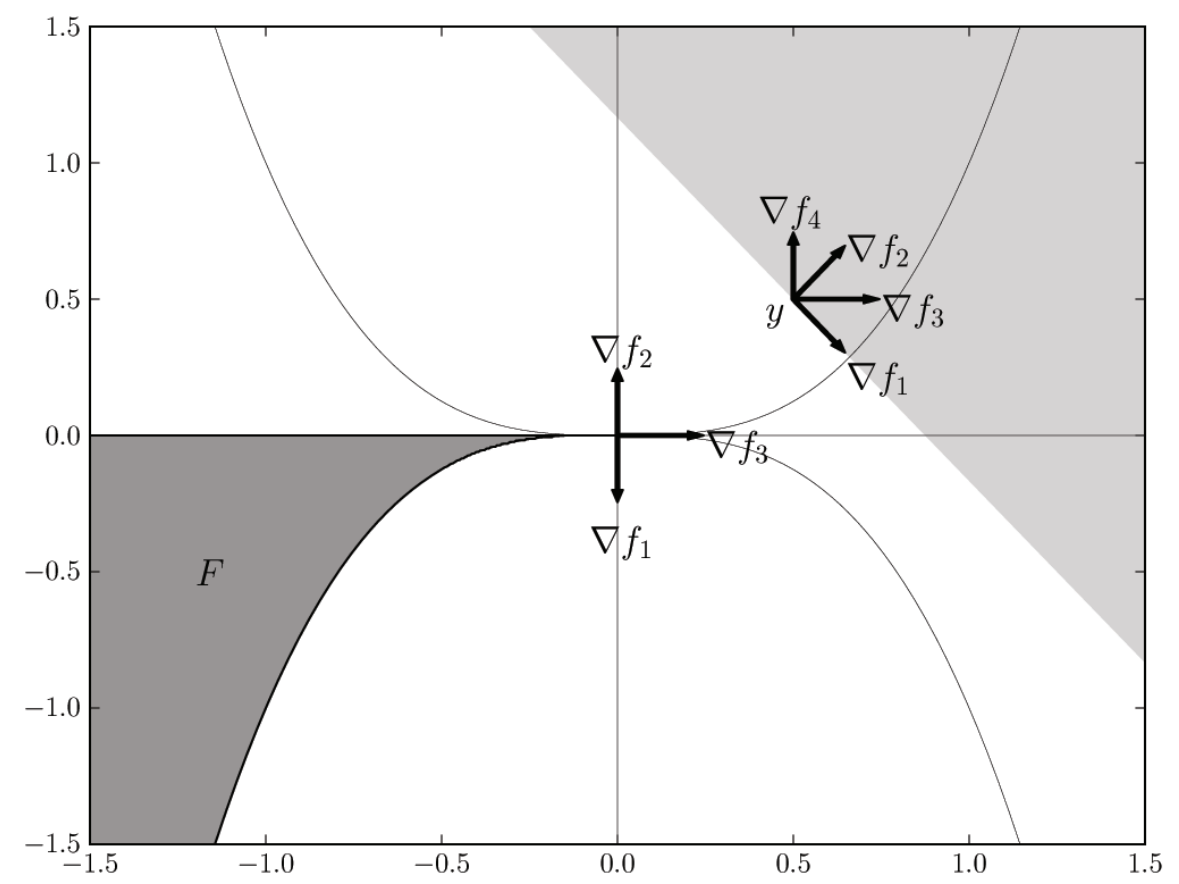

FIG. 3.2. Consider $F=\left\{\left(x_{1}, x_{2}\right) \in \mathbb{R}^{2} \mid f_{1}\left(x_{1}, x_{2}\right)=x_{1}^{3}-x_{2} \leq 0, f_{2}\left(x_{1}, x_{2}\right)=x_{1}^{3}+x_{2} \leq\right.$ $\left.0, f_{3}\left(x_{1}, x_{2}\right)=x_{1} \leq 0, f_{4}\left(x_{1}, x_{2}\right)=x_{2}^{3} \leq 0\right\}$ at the origin. Then, $\operatorname{span}_{+}(\{1\},\{3\} ; G f(y))$ is the light gray semispace and contains all the gradients. On the other hand, $\nabla f_{4}(y) \notin \operatorname{span}_{+}(\{2\},\{3\} ; G f(y))$ whenever $y \neq 0$. 
$\left\{\lambda^{k}\right\} \subset \mathbb{R}^{m},\left\{\mu^{k}\right\} \subset \mathbb{R}^{p}, \mu^{k} \geq 0$, such that

$$
\nabla f_{0}\left(x^{k}\right)+\sum_{i \in \mathcal{I}} \lambda_{i}^{k} \nabla f_{i}\left(x^{k}\right)+\sum_{j \in \mathcal{A}(x)} \mu_{j}^{k} \nabla f_{m+j}\left(x^{k}\right)=\epsilon^{k} .
$$

In this case, we may also say that $x$ is an AKKT point.

It is well known from [4] that if $x$ is a local minimum, then it must be an AKKT point. Therefore, to prove that CPG is a CQ, all we need to show is that if CPG holds at an AKKT point, then it has to be a KKT point. Another important property is that many methods for nonlinear optimization are guaranteed to converge to AKKT points. Hence, it will be a corollary of Theorem 3.3 below that if one of such algorithms generates a sequence converging to a point where CPG holds, then such a point has to be a KKT point. This will be the main tool used in section 5.2, where we describe applications of CPG to the convergence analysis of nonlinear optimization methods.

THEOREM 3.3. Let $x$ be a feasible point of (NOP) that satisfies the AKKT condition. If $x$ also satisfies $C P G$, then $x$ is a KKT point.

Proof. Let $x^{k}, \epsilon^{k}, \lambda^{k}$, and $\mu^{k}$ be the sequences given by the AKKT condition. Let $\left(\mathcal{I}^{\prime}, \mathcal{J}_{+}\right)$be the positively linearly independent spanning pair given by CPG. Then, for each sufficiently large $k$ there must be $\bar{\lambda}_{i}^{k}, i \in \mathcal{I}^{\prime}$, and $\bar{\mu}_{j}^{k} \geq 0, j \in \mathcal{J}_{+}$, such that

$$
\nabla f_{0}\left(x^{k}\right)+\sum_{i \in \mathcal{I}^{\prime}} \bar{\lambda}_{i}^{k} \nabla f_{i}\left(x^{k}\right)+\sum_{j \in \mathcal{J}_{+}} \bar{\mu}_{j}^{k} \nabla f_{j}\left(x^{k}\right)=\epsilon^{k}
$$

Define $M_{k}=\max \left\{\left|\bar{\lambda}_{i}^{k}\right|, i \in \mathcal{I}^{\prime} ; \bar{\mu}_{j}^{k}, j \in \mathcal{J}_{+}\right\}$. There are two possibilities:

1. If $M_{k}$ has a bounded subsequence, we can assume, by possibly extracting a further subsequence, that for all $i \in \mathcal{I}^{\prime}$ and $j \in \mathcal{J}_{+}$the subsequences of $\bar{\lambda}_{i}^{k}$ and $\bar{\mu}_{j}^{k}$ have limits $\bar{\lambda}_{i}^{*}$ and $\bar{\mu}_{j}^{*} \geq 0$, respectively. Then, taking the limit at (3.2), we arrive at

$$
\nabla f_{0}(x)+\sum_{i \in \mathcal{I}^{\prime}} \bar{\lambda}_{i}^{*} \nabla f_{i}(x)+\sum_{j \in \mathcal{J}_{+}} \bar{\mu}_{j}^{*} \nabla f_{j}(x)=0 .
$$

As

$$
\sum_{i \in \mathcal{I}^{\prime}} \bar{\lambda}_{i}^{*} \nabla f_{i}(x)+\sum_{j \in \mathcal{J}_{+}} \bar{\mu}_{j}^{*} \nabla f_{j}\left(x^{k}\right) \in \operatorname{span}_{+}(\mathcal{I}, \mathcal{J} ; G f(x)),
$$

we see that $x$ is KKT.

2. If $M_{k} \rightarrow \infty$, we can divide (3.2) by $M_{k}$ for $k$ large enough and get

$$
\frac{1}{M_{k}} \nabla f_{0}\left(x^{k}\right)+\sum_{i \in \mathcal{I}^{\prime}} \frac{\bar{\lambda}_{i}^{k}}{M_{k}} \nabla f_{i}\left(x^{k}\right)+\sum_{j \in \mathcal{J}_{+}} \frac{\bar{\mu}_{j}^{k}}{M_{k}} \nabla f_{j}\left(x^{k}\right)=\frac{\epsilon^{k}}{M_{k}} .
$$

We can then take the limit in the equation above and derive a contradiction to the fact that $\left(\mathcal{I}^{\prime}, \mathcal{J}_{+}\right)$is positively linearly independent.

Corollary 3.4. The CPG condition is a $C Q$.

4. Relation with other constraint qualifications. Now that we know that $\mathrm{CPG}$ is a $\mathrm{CQ}$, it is natural to ask what its relation is to other CQs in the literature. Let us start with its relation to RCRCQ, which is naturally connected to CRSC as defined in the introduction. 
THEOREM 4.1. The constant rank of the subspace component (CRSC) condition implies CPG.

Proof. Let $\left(\mathcal{I}^{\prime}, \mathcal{J}_{+}\right)$be a positively linearly independent spanning pair of the cone $\operatorname{span}_{+}(\mathcal{I}, \mathcal{J} ; G f(x))$. It suffices for us to show that in a neighborhood of $x$, $\nabla f_{\ell}(y) \in \operatorname{span}\left\{\nabla f_{i}(y) \mid i \in \mathcal{I}^{\prime}\right\}$ for all $\ell \in \mathcal{I} \cup \mathcal{J}_{-}$. We know from Lemma 2.5 that $\mathcal{I}^{\prime}$ is the set of indexes of a basis for $\operatorname{span}\left\{\nabla f_{i}(x) \mid i \in \mathcal{I} \cup \mathcal{J}_{-}\right\}$. As the rank of $\left\{\nabla f_{i}(y) \mid i \in \mathcal{I} \cup \mathcal{J}_{-}\right\}$remains constant in a neighborhood of $x$, this basis has to remain a basis in a (possibly smaller) neighborhood of $x$.

Note that, in particular, the theorem above shows that RCRCQ implies CPG, as RCRCQ implies CRSC. Moreover, CRSC successfully eliminates the need to test all subsets involving the gradients of active inequality constraints. CRSC simplified RCRCQ as the latter simplified Janin's CQ for feasible sets with only equality constraints.

Another CQ in the same family is RCPLD, which is related to RCRCQ as CPLD is related to the original constant rank. That is, RCPLD trades the constant rank assumption in RCRCQ by the local preservation of positive linear dependence, a weaker condition.

Definition 4.2. Let $x$ be a feasible point of (NOP). Let $\tilde{\mathcal{I}}$ be the set of indexes of a basis of $\operatorname{span}\left\{\nabla f_{i}(x) \mid i \in \mathcal{I}\right\}$. We say that $x$ satisfies RCPLD if there is a neighborhood $N(x)$ of $x$, where

1. for all $y \in N(x),\left\{\nabla f_{i}(y) \mid i \in \mathcal{I}\right\}$ has constant rank;

2. for all subsets of indexes of active inequality constraints $\tilde{\mathcal{J}} \subset \mathcal{A}(x)$, if $(\tilde{\mathcal{I}}, \tilde{\mathcal{J}})$ is positively linearly dependent at $x$, then it remains positively linearly dependent (or, equivalently, linearly dependent) in $N(x)$.

We prove below that the RCPLD, just as RCRCQ, also locally preserves the rank of $\left\{\nabla f_{i}(y) \mid i \in \mathcal{I} \cup \mathcal{J}_{-}\right\}$; that is, it also implies CRSC.

THEOREM 4.3. RCPLD implies CRSC.

Proof. From Corollary 2.7 we know that if $j \in \mathcal{J}_{-}$, then $-\nabla f_{j}(x)$ can be positively spanned by the other vectors in the pair $\left(\mathcal{I}, \mathcal{J}_{-}\right)$. By the definition of RCPLD, this fact remains true in $N(x)$, and hence $\operatorname{span}_{+}\left(\mathcal{I}, \mathcal{J}_{-} ; G f(y)\right)$ is actually a subspace for all $y \in N(x)$. What we want to show is that these subspaces have the same dimension as the subspace $\operatorname{span}_{+}\left(\mathcal{I}, \mathcal{J}_{-} ; G f(x)\right)$ in a smaller neighborhood of $x$.

Let $\tilde{N}(x)$ be a neighborhood of $x$ contained in $N(x)$ such that the dimension of $\operatorname{span}_{+}\left(\mathcal{I}, \mathcal{J}_{-} ; G f(y)\right)$ is greater than or equal to the dimension of $\operatorname{span}_{+}\left(\mathcal{I}, \mathcal{J}_{-} ; G f(x)\right)$, which exists as linear independence is preserved locally. We thus need to show that the dimension cannot increase, remaining constant.

We start by noting that if $\tilde{\mathcal{I}}$ is as in the definition of RCPLD, then for all $y \in \tilde{N}(x)$, $\operatorname{span}_{+}\left(\mathcal{I}, \mathcal{J}_{-} ; G f(y)\right)=\operatorname{span}_{+}\left(\tilde{\mathcal{I}}, \mathcal{J}_{-} ; G f(y)\right)$. Let $\tilde{m}=\# \tilde{\mathcal{I}}$, the cardinality of $\tilde{\mathcal{I}}$, $n_{-}=\# \mathcal{J}_{-}, \tilde{\mathcal{I}}=\left\{i_{1}, i_{2}, \ldots, i_{\tilde{m}}\right\}$, and $\mathcal{J}_{-}=\left\{j_{1}, j_{2}, \ldots, j_{n_{-}}\right\}$. Define

$$
\begin{gathered}
v_{l}(y) \stackrel{\text { def }}{=} \nabla f_{i_{l}}(y), \quad l=1, \ldots, \tilde{m}, \\
v_{\tilde{m}+l}(y) \stackrel{\text { def }}{=}-\nabla f_{i_{l}}(y), \quad l=1, \ldots, \tilde{m}, \\
v_{2 \tilde{m}+l}(y) \stackrel{\text { def }}{=} \nabla f_{j_{l}}(y), \quad l=1, \ldots, n_{-},
\end{gathered}
$$

and define the set $\mathcal{A} \stackrel{\text { def }}{=}\left\{1,2, \ldots, 2 \tilde{m}+n_{-}\right\}$.

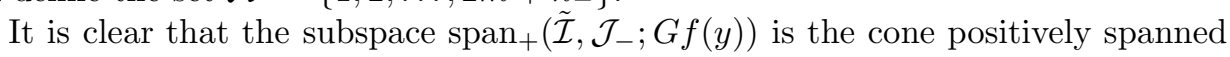
by $A(y) \stackrel{\text { def }}{=}\left\{v_{l}(y) \mid l \in \mathcal{A}\right\}$; in particular, it is linearly spanned by $A(y)$. Moreover, if a subset of vectors in $A(x)$ is linearly dependent using only nonnegative weights, 
then RCPLD asserts that, for $y \in \tilde{N}(y)$, the respective vectors in $A(y)$ remain linearly dependent using only nonnegative weights.

Now let $v_{l^{\prime}}(x)$ be a vector in $A(x)$ that can be positively spanned by the other vectors in $A(x)$. Then $A(x) \backslash\left\{v_{l^{\prime}}(x)\right\}$ still positively spans the same space. Moreover, as $A(x)$ spans the space positively, we know that $-v_{l^{\prime}}(x)$ can be written as a positive combination of the remaining vectors in $A(x)$; that is, there are $\alpha_{l} \geq 0$ such that

$$
-v_{l^{\prime}}(x)=\sum_{l \in \mathcal{A} \backslash\left\{l^{\prime}\right\}} \alpha_{l} v_{l}(x) .
$$

Using Carathodory's lemma [8, Exercise B.1.7], we can reduce this sum to a subset $\mathcal{A}^{\prime} \subset \mathcal{A} \backslash\left\{l^{\prime}\right\}$ such that the respective $\alpha_{l}>0$ and the vectors $v_{l}(x), l \in \mathcal{A}^{\prime}$, are positively linearly independent. As RCPLD holds, this fact remains true in $\tilde{N}(x)$, and hence the vector $v_{l^{\prime}}(y)$ is not necessary to describe the subspace linearly spanned by $A(y)$.

Hence, if we iteratively delete from $A(x)$ vectors that can be positively spanned by the other vectors in the set, delete from $\mathcal{A}$ the respective index, and call $\tilde{\mathcal{A}}$ the final index set, we can see that

1. the subspace $\operatorname{span}_{+}\left(\mathcal{I}, \mathcal{J}_{-} ; G f(x)\right)$ is the cone positively generated by the vectors in $\tilde{A}(x) \stackrel{\text { def }}{=}\left\{v_{l}(x) \mid l \in \tilde{\mathcal{A}}\right\}$, and $\tilde{A}(x)$ is a positive basis for this subspace [39];

2. for all $y \in \tilde{N}(x)$, the subspace $\operatorname{span}_{+}\left(\mathcal{I}, \mathcal{J}_{-} ; G f(y)\right)$ is the subspace linearly spanned by $\tilde{A}(y) \stackrel{\text { def }}{=}\left\{v_{l}(y) \mid l \in \tilde{\mathcal{A}}\right\}$.

We can then apply Lemma 6 from [39] to $\tilde{A}(x)$ to see that there is a partition of the index set $\tilde{\mathcal{A}}$ into $p$ pairwise disjoint subsets $\tilde{\mathcal{A}}_{1} \cup \cdots \cup \tilde{\mathcal{A}}_{p}$ such that the positive cone generated by $\left\{v_{l}(x) \mid l \in \tilde{\mathcal{A}}_{1} \cup \cdots \cup \tilde{\mathcal{A}}_{p^{\prime}}\right\}$ is a linear subspace of dimension $\left(\sum_{k=1}^{p^{\prime}} \# \tilde{A}_{k}\right)-p^{\prime}$ for each $p^{\prime}=1,2, \ldots, p$. In particular, the dimension of the space positively spanned by $\tilde{A}(x)$ is $\# \tilde{\mathcal{A}}-p$.

Take $p^{\prime}=1$. The partition properties ensure that if we delete a vector $v_{l_{1}}(x)$ from $\left\{v_{l}(x) \mid l \in \tilde{\mathcal{A}}_{1}\right\}$, then the remaining ones are linearly independent. Moreover, $v_{l_{1}}(x)$ not only is linearly dependent with the remaining ones, it is positively linearly dependent, as its negative has to be positively spanned by the others. This positive linear dependence is preserved by RCPLD, and hence the space linearly spanned by $\left\{v_{l}(y) \mid l \in \tilde{\mathcal{A}}_{1}\right\}$ is the same as the space linearly spanned by $\left\{v_{l}(y) \mid l \in \tilde{\mathcal{A}}_{1}, l \neq l_{1}\right\}$ for $y \in \tilde{N}(x)$.

Now take $p^{\prime}=2$. There is vector $v_{l_{2}}(x) \in\left\{v_{l}(x) \mid l \in \tilde{\mathcal{A}}_{2}\right\}$ such that $\left\{v_{l}(x) \mid\right.$ $\left.l \in \tilde{\mathcal{A}}_{1} \cup \tilde{\mathcal{A}}_{2}, l \notin\left\{l_{1}, l_{2}\right\}\right\}$ is a basis of the subspace positively spanned by $\left\{v_{l}(x) \mid\right.$ $\left.l \in \tilde{\mathcal{A}}_{1} \cup \tilde{\mathcal{A}}_{2}\right\}$. As this space is positively spanned, we can see that there must be nonnegative coefficients $\alpha_{l}$ such that

$$
-v_{l_{2}}(x)=\sum_{l \in \tilde{\mathcal{A}}_{1} \cup \tilde{\mathcal{A}}_{2} \backslash\left\{l_{2}\right\}} \alpha_{l} v_{l}(x) .
$$

Again using Carathodory's lemma, we can see that RCPLD ensures that for $y \in$ $\tilde{N}(y)$ the vector $v_{l_{2}}(y)$ is not necessary to describe the subspace linearly spanned by $\left\{v_{l}(y) \mid l \in \tilde{\mathcal{A}}_{1} \cup \tilde{\mathcal{A}}_{2}\right\}$. That is, for $y \in \tilde{N}(x)$, the subspace linearly spanned by $\left\{v_{l}(y) \mid l \in \tilde{\mathcal{A}}_{1} \cup \tilde{\mathcal{A}}_{2}\right\}$ is the same as the one linearly spanned by $\left\{v_{l}(y) \mid l \in \tilde{\mathcal{A}}_{1} \cup \tilde{\mathcal{A}}_{2}, l \neq\right.$ $\left.l_{2}\right\}$, which in turn is the same as the one linearly spanned by $\left\{v_{l}(y) \mid l \in \tilde{\mathcal{A}}_{1} \cup \tilde{\mathcal{A}}_{2}, l \notin\right.$ $\left.\left\{l_{1},, l_{2}\right\}\right\}$. 
This process can be carried on $p$ times, and at the end we conclude that for all $y \in \tilde{N}(x)$ there are $p$ vectors in $\tilde{A}(y)$ that are not necessary to describe its linearly spanned set, which in turn is $\operatorname{span}_{+}\left(\mathcal{I}, \mathcal{J}_{-} ; G f(y)\right)$. Hence, the dimension of $\operatorname{span}_{+}\left(\mathcal{I}, \mathcal{J}_{-} ; G f(y)\right)$ is less than or equal to $\# \tilde{A}(y)-p=\# \tilde{\mathcal{A}}-p$. This last value is the dimension of the space linearly spanned by $\tilde{A}(x)$, namely, $\operatorname{span}_{+}\left(\mathcal{I}, \mathcal{J}_{-}\right.$; $G f(x))$.

Note that the CRSC condition is not equivalent to the CPG condition. Actually, consider once again the feasible set pictured in Figure 3.1:

$$
\left\{\left(x_{1}, x_{2}\right) \in \mathbb{R}^{2} \mid x_{1}^{3}-x_{2} \leq 0, x_{1}^{3}+x_{2} \leq 0, x_{1} \leq 0\right\} .
$$

Then, at the origin $\mathcal{J}_{-}=\{1,2\}$ and the rank of $\left\{\nabla f_{1}(0), \nabla f_{2}(0)\right\}$ is 1 . On the other hand, for any $y \neq 0$, the rank increases while CPG holds. In particular, CPG is a proper generalization of RCPLD.

Finally, let us show that CPG implies Abadie's CQ. In order to achieve this we start with a result that can be directly deduced from the proof of Theorem 4.3.1 in [7].

LEMma 4.4. Let $x$ be a feasible point of (NOP) that conforms to the MFCQ; i.e., the set $\left\{\nabla f_{i}(x) \mid i \in \mathcal{I}\right\}$ is linearly independent and there is a direction $0 \neq d \in \mathbb{R}^{n}$ such that

$$
\nabla f_{i}(x)^{\prime} d=0, \quad i \in \mathcal{I}, \quad \nabla f_{i}(x)^{\prime} d<0, \quad i \in \mathcal{J} .
$$

Then, there is a scalar $T>0$ and a continuously differentiable arc $\alpha:[0, T] \rightarrow \mathbb{R}^{n}$ such that

$$
\begin{aligned}
\alpha(0) & =x, \\
\dot{\alpha}(0) & =d, \\
f_{i}(\alpha(t)) & =0 \quad \forall t \in[0, T], \quad i \in \mathcal{I}, \\
\nabla f_{i}(\alpha(t))^{\prime} \dot{\alpha}(t) & =0 \quad \forall t \in[0, T], \quad i \in \mathcal{I}, \\
f_{j}(\alpha(t)) & <0 \quad \forall t \in(0, T], \quad j \in \mathcal{J}, \\
\nabla f_{j}(\alpha(t))^{\prime} \dot{\alpha}(t) & <0 \quad \forall t \in[0, T], \quad j \in \mathcal{J} .
\end{aligned}
$$

Now we use the lemma above to find special differentiable arcs that move inward the feasible set under CPG.

LEMma 4.5. Let $x$ be a feasible point for (NOP), where CPG holds, and let $\left(\mathcal{I}^{\prime}, \mathcal{J}_{+}\right)$be the associated positively linearly independent spanning pair. Then there exists $0 \neq d \in \mathbb{R}^{n}$ such that

$$
\nabla f_{i}(\bar{x})^{\prime} d=0, \quad i \in \mathcal{I}^{\prime}, \quad \nabla f_{j}(\bar{x})^{\prime} d<0, \quad j \in \mathcal{J}_{+} .
$$

Moreover, for any such $d$, there is a scalar $T>0$ and a continuously differentiable arc $\alpha:[0, T] \rightarrow \mathbb{R}^{n}$ such that

$$
\begin{aligned}
\alpha(0) & =\bar{x} \\
\dot{\alpha}(0) & =d, \\
f_{i}(\alpha(t)) & =0 \quad \forall t \in[0, T], i \in \mathcal{I}, \\
f_{j}(\alpha(t)) & \leq 0 \quad \forall t \in(0, T], \quad j \in \mathcal{J} .
\end{aligned}
$$

Proof. As $\left(\mathcal{I}^{\prime}, \mathcal{J}_{+}\right)$is positively linearly independent, the feasible set described by

$$
\left\{x \mid f_{i}(x)=0, i \in \mathcal{I}^{\prime}, f_{j}(x) \leq 0, j \in \mathcal{J}_{+}\right\}
$$


conforms to the MFCQ at $x$. Therefore the desired direction $d$ exists.

Let $\alpha:[0, T] \mapsto \mathbb{R}^{n}$ be the curve given by Lemma 4.4, and take $0<T^{\prime} \leq T$ to ensure that for all $t \in\left[0, T^{\prime}\right], \alpha(t) \in N(x)$, where $N(x)$ is the neighborhood of $x$ given by CPG. We already know that (4.9)-(4.10) hold for constraints with indexes in $\mathcal{I}^{\prime} \cup \mathcal{J}_{+}$; hence, all we need to show is that they also hold for $\ell \in\left(\mathcal{I} \cup \mathcal{J}_{-}\right) \backslash \mathcal{I}^{\prime}$.

Fix such an index $\ell$. We know that $\nabla f_{\ell}(y)$ belongs to $\operatorname{span}_{+}\left(\mathcal{I}^{\prime}, \mathcal{J}_{+} ; G f(y)\right)$ for all $y \in N(x)$. That is, there are scalars $\lambda_{i}(y), i \in \mathcal{I}^{\prime}$, and $\mu_{j}(y) \geq 0, j \in \mathcal{J}_{+}$, such that

$$
\nabla f_{\ell}(y)=\sum_{i \in \mathcal{I}^{\prime}} \lambda_{i}(y) \nabla f_{i}(y)+\sum_{j \in \mathcal{J}_{+}} \mu_{j}(y) \nabla f_{j}(y)
$$

Define $\varphi_{\ell}(t)=f_{\ell}(\alpha(t))$. It follows that

$$
\begin{aligned}
\varphi_{\ell}^{\prime}(t) & =\nabla f_{\ell}(\alpha(t))^{\prime} \dot{\alpha}(t) \\
& =\sum_{i \in \mathcal{I}^{\prime}} \lambda_{i}(\alpha(t)) \nabla f_{i}(x)^{\prime} \dot{\alpha}(t)+\sum_{j \in \mathcal{J}_{+}} \mu_{j}(\alpha(t)) \nabla f_{j}(\alpha(t))^{\prime} \dot{\alpha}(t) \\
& \leq 0
\end{aligned}
$$

The last inequality follows from the sign structure given in Lemma 4.4. Hence, if $\ell$ is associated with an inequality constraint, (4.10) is proved. On the other hand, if $\ell$ is associated with an equality constraint, we know that $-\nabla f_{\ell}(x)$ also belongs to $\operatorname{span}_{+}\left(\mathcal{I}^{\prime}, \mathcal{J}_{+} ; G f(y)\right)$ for $y \in N(x)$. We can then proceed as above to see that

$$
-\varphi_{\ell}^{\prime}(t) \leq 0
$$

And hence we conclude that (4.9) holds.

We are ready to show that CPG implies Abadie's CQ.

Theorem 4.6. $C P G C Q$ at $x$ implies Abadie's $C Q$ at $x$.

Proof. Let $d$ be the direction given in Lemma 4.5. Given

$$
\bar{d} \in\left\{h \mid \nabla f_{i}(\bar{x})^{\prime} h=0, i \in \mathcal{I}, \nabla f_{j}(\bar{x})^{\prime} h \leq 0, j \in \mathcal{J}\right\}
$$

we need to show that $\bar{d}$ belongs to the tangent cone of the feasible set at $x$ (Definition 1.1).

Clearly, for arbitrary $\epsilon>0, \bar{d}+\epsilon d$ inherits from $d$ the properties required to apply Lemma 4.5. Hence there is a $T>0$ and a feasible continuously differentiable arc $\alpha:[0, T] \rightarrow \mathbb{R}^{n}$ such that

$$
\alpha(0)=x, \quad \dot{\alpha}(0)=\bar{d}+\epsilon d .
$$

It follows that $\bar{d}+\epsilon d$ belongs to the tangent cone of the feasible set at $x$. Moreover, as this cone is closed, $\bar{d}$ also belongs to it.

In Figure 4.1, we show a complete diagram displaying the relations of CRSC and CPG with other CQs, including pseudo- and quasi normality, whose definitions can be found in [8]. To obtain all the relations, we used the results presented here together with the examples and results from [5]. 


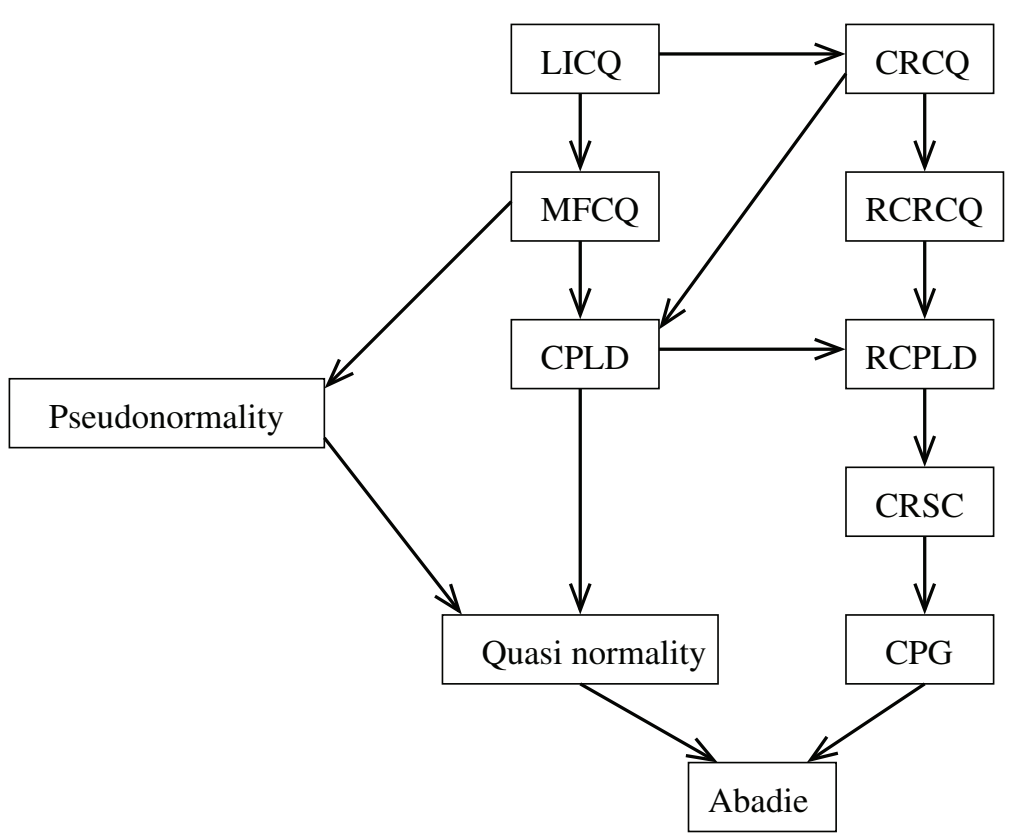

FIG. 4.1. Complete diagram showing the relations of CRSC and CPG with other well-known $C Q s$. An arrow between two CQs means that one is strictly stronger than the other, while conditions that are not connected by a directed path are independent from each other. Note that pseudonormality does not imply CPG, as Example 3 in [5] shows.

\section{Applications of CRSC and CPG.}

5.1. Error bound. One interesting question about a $C Q$ is whether it implies an error bound. That is, we ask whether it is possible to use a natural measure of infeasibility to estimate the distance to the feasible set $F$ close to a feasible point $x$.

DEFINITION 5.1 (see [41]). We say that an error bound holds in a neighborhood $N(x)$ of a feasible point $x \in F$ if there exists $\alpha>0$ such that for every $y \in N(x)$

$$
\begin{aligned}
\min _{z \in F}\|z-y\| \leq \alpha \max \left\{\left|f_{i}(y)\right|, i=1, \ldots, m ;\right. \\
\left.\max \left\{0, f_{j}(y)\right\}, j=m+1, \ldots, m+p\right\} .
\end{aligned}
$$

This property is valid for many CQs, and in particular, for weak ones such as RCPLD [5] and quasi normality [34]. It has important theoretical and algorithmic implications; see, for example, [36, 41].

Unfortunately, such a property does not hold for CPG, as the example in Figure 3.2 shows. In this case, there is no error bound around the origin. To see this, consider the sequence $x^{k}=(-\sqrt[3]{1 / k}, 1 / k)$. The distance of $x^{k}$ to the feasible set is exactly $1 / k$, while the infeasibility measure is $1 / k^{3}$. Note that, by increasing the exponent that appears in the definition of the violated constraint $f_{4}$ and adapting the sequence accordingly, it is possible to make the infeasibility converge to zero as fast as $1 / k^{2 p+1}$, for any positive integer $p$, while the distance to the feasible set remains $1 / k$. On the other hand, we will now show that the CRSC CQ is enough to ensure the validity of an error bound. 
Throughout this subsection, we use $x$ to denote a fixed feasible point that verifies CRSC, and we denote by $B \subset \mathcal{I}$ an index set such that $\left\{\nabla f_{i}(x)\right\}_{i \in B}$ is a basis of $\operatorname{span}\left\{\nabla f_{i}(x)\right\}_{i \in \mathcal{I}}$. We will also need to compute the sets $\mathcal{J}, \mathcal{J}_{-}$, and $\mathcal{J}_{+}$that appear in the definition of CRSC and CPG in points that are not $x$. Hence, given a feasible point $y$, we will use the following definitions:

$$
\begin{aligned}
& \mathcal{J}(y) \stackrel{\text { def }}{=} \mathcal{A}(y), \\
& \mathcal{J}_{-}(y) \stackrel{\text { def }}{=}\left\{j \in \mathcal{J}(x) \mid-\nabla f_{j}(y) \in \mathcal{F}(x)^{\circ}\right\}, \\
& \mathcal{J}_{+}(y) \stackrel{\text { def }}{=} \mathcal{J}(y) \backslash \mathcal{J}_{-}(y) .
\end{aligned}
$$

Using this notation, CRSC ensures that the rank of the vectors $\left\{\nabla f_{i}(y) \mid i \in B \cup\right.$ $\left.\mathcal{J}_{-}(x)\right\}$ is constant in a neighborhood of $x$. Moreover, if $K$ is an index set, let us denote by $f_{K}$ the function whose components are the $f_{i}$ such that $i$ belongs to $K$.

We start the analysis of CRSC with a technical result.

LEMma 5.2. Let $x$ be a feasible point that verifies CRSC. Then, there exist scalars $\lambda_{i}, i \in B$, and $\mu_{j}$ with $\mu_{j}>0$ for all $j \in \mathcal{J}_{-}(x)$ such that

$$
\sum_{i \in B} \lambda_{i} \nabla f_{i}(x)+\sum_{j \in \mathcal{J}_{-}(x)} \mu_{j} \nabla f_{j}(x)=0 .
$$

Proof. We know that for any index $l \in \mathcal{J}_{-}(x)$ there exist scalars $\lambda_{i}^{l}, i \in B$, and $\mu_{j}^{l}$ with $\mu_{j}^{l} \geq 0$ such that

$$
-\nabla f_{l}(x)=\sum_{i \in B} \lambda_{i}^{l} \nabla f_{i}(x)+\sum_{j \in \mathcal{J}_{-}(x)} \mu_{j}^{l} \nabla f_{j}(x) .
$$

Thus, adding for all $l \in \mathcal{J}_{-}(x)$ both sides of the above equality and rearranging the resulting terms, we get

$$
\sum_{i \in B} \gamma_{i} \nabla f_{i}(x)+\sum_{j \in \mathcal{J}_{-}(x)} \theta_{j} \nabla f_{j}(x)=0,
$$

where $\gamma_{i}=\sum_{l \in \mathcal{J}_{-}(x)} \lambda_{i}^{l}$ and $\theta_{j}=1+\sum_{l \in \mathcal{J}_{-}(x)} \mu_{j}^{l} \geq 1>0$.

The next lemma extends an important result from Lu [23] for CRCQ to CRSC. Namely, it shows that the constraints $f_{j}$ with $j \in \mathcal{J}_{-}(x)$ are actually equality constraints under the disguise of inequalities.

Lemma 5.3. Let $x$ be a feasible point that verifies CRSC. Then, there exists a neighborhood $N(x)$ of $x$ such that, for every $i \in \mathcal{J}_{-}(x), f_{i}(y)=0$ for all feasible points $y \in N(x)$.

Proof. From the previous lemma there exist scalars $\lambda_{i}, i \in B$, and $\mu_{j}>0$ for all $j \in \mathcal{J}_{-}(x)$ such that (5.1) holds.

Since the rank of the vectors $\left\{\nabla f_{i}(y) \mid i \in B \cup \mathcal{J}_{-}(x)\right\}$ is constant for $y$ in a neighborhood of $x$, we can use [23, Proposition 1], defining the index sets $K$ and $J_{0}$ in [23] as the sets $\mathcal{J}_{-}(x)$ and $B$, respectively, to complete the proof.

Observe that, even though the hypothesis considered in [23, Proposition 1] is the CRCQ, the proof is obtained by applying the respective Lemma 1 , where only the constant rank of the gradients in $K=\mathcal{J}_{-}(x)$ and $J_{0}=B$ is used. Actually, such a lemma can be viewed as a variation of the constant rank theorem [25] where only the rank of all gradients has to remain constant. 
Now we are ready to show that the CRSC condition is preserved locally. That is, if it holds at a feasible point $x$, it must hold at all feasible points in a neighborhood of $x$. We start by showing that the index set $\mathcal{J}_{-}$is stable locally.

Lemma 5.4. Let $x$ be a feasible point that verifies CRSC. Then there exists a neighborhood $N(x)$ of $x$ such that $\mathcal{J}_{-}(y)=\mathcal{J}_{-}(x)$ for all feasible points $y \in N(x)$.

Proof. From Lemma 5.2 we know that there exist scalars $\lambda_{i}, i \in B$, and $\mu_{j}$ with $\mu_{j}>0$ for all $j \in \mathcal{J}_{-}(x)$ such that (5.1) holds.

Let us take a subset $\widehat{\mathcal{J}} \subset \mathcal{J}_{-}(x)$ such that the set of gradients $\left\{\nabla f_{i}(x)\right\}_{i \in B \cup \widehat{\mathcal{J}}}$ is a basis of $\operatorname{span}\left\{\nabla f_{i}(x)\right\}_{i \in \mathcal{I} \cup \mathcal{J}_{-}(x)}$. Clearly the set of gradients

$$
\left\{\nabla f_{i}(x)\right\}_{i \in B \cup \widehat{\mathcal{J}}}
$$

is linearly independent.

Define the function

$$
h(y)=-\sum_{j \in \mathcal{J}_{-}(x) \backslash \widehat{\mathcal{J}}} \mu_{j} f_{j}(y)
$$

and let us consider a new feasible set $F^{h}$ adding to the original feasible set $F$ the equality constraint $h(y)=0$, which is locally redundant by Lemma 5.3. Let us define $\mathcal{J}_{-}^{h}(\cdot)$ analogously for $F^{h}$ as we define $\mathcal{J}_{-}(\cdot)$ for the original feasible set $F$. Thus, we have

1. $h(y)=0$ for all $y \in F \cap N(x)$;

2. $\nabla h(x) \in \mathcal{J}_{-}^{h}(x)$;

3 . the set of gradients

$$
\left\{\nabla h(y), \nabla f_{i}(y) \mid i \in B \cup \widehat{\mathcal{J}}\right\}
$$

has constant rank in a neighborhood of $x$, as $\nabla h$ is a combination of $\nabla f_{i}, i \notin$ $B \cup \widehat{\mathcal{J}}$, and each of the later gradients are generated by $\nabla f_{i}, i \in B \cup \mathcal{\mathcal { J }}$, by CRSC.

Recalling (5.1), we get

$$
\nabla h(x)=-\sum_{j \in \mathcal{J}_{-}(x) \backslash \widehat{\mathcal{J}}} \mu_{j} \nabla f_{j}(x)=\sum_{i \in B} \lambda_{i} \nabla f_{i}(x)+\sum_{j \in \widehat{\mathcal{J}}} \mu_{j} \nabla f_{j}(x),
$$

and therefore, using conditions (5.2)-(5.3), we can apply [23, Corollary 1] to obtain neighborhoods $N(x)$ of $x, Z$ of $\left(f_{B}(x), f_{\widehat{\mathcal{J}}}(x)\right)$, with $Z$ being convex, and a continuously differentiable function $g: Z \rightarrow \mathbb{R}$ such that

$$
h(x)=g\left(f_{B}(x), f_{\widehat{\mathcal{J}}}(x)\right)
$$

and, for every $z \in Z$,

$$
\begin{aligned}
& \operatorname{sgn}\left(\frac{\partial g}{\partial z_{i}}(z)\right)=\operatorname{sgn}\left(\lambda_{i}\right) \quad \forall i \in B, \\
& \operatorname{sgn}\left(\frac{\partial g}{\partial z_{i}}(z)\right)=\operatorname{sgn}\left(\mu_{i}\right) \quad \forall i \in \widehat{\mathcal{J}} .
\end{aligned}
$$


Thus, by the definition of $h$ and (5.5), it follows that for all $y \in F$ in a neighborhood of $x$

$$
\begin{aligned}
\nabla h(y) & =-\sum_{j \in \mathcal{J}_{-}(x) \backslash \widehat{\mathcal{J}}} \mu_{j} \nabla f_{j}(y) \\
& =\sum_{i \in B} \frac{\partial g}{\partial z_{i}}\left(f_{B}(y), f_{\widehat{\mathcal{J}}}(y)\right) \nabla f_{i}(y)+\sum_{j \in \widehat{\mathcal{J}}} \frac{\partial g}{\partial z_{j}}\left(f_{B}(y), f_{\widehat{\mathcal{J}}}(y)\right) \nabla f_{j}(y) .
\end{aligned}
$$

Using (5.6), (5.7), and (5.9), there are scalars $\gamma_{i}(y)=\frac{\partial g}{\partial z_{i}}\left(f_{B}(y), f_{\widehat{\mathcal{J}}}(y)\right)$ and $\theta_{j}(y)=$ $\frac{\partial g}{\partial z_{j}}\left(f_{B}(y), f_{\widehat{\mathcal{J}}}(y)\right)>0$ such that

$$
\sum_{j \in \mathcal{J}_{-}(x) \backslash \widehat{\mathcal{J}}} \mu_{j} \nabla f_{j}(y)+\sum_{i \in B} \gamma_{i}(y) \nabla f_{i}(y)+\sum_{j \in \widehat{\mathcal{J}}} \theta_{j}(y) \nabla f_{j}(y)=0 .
$$

From the last expression, Lemma 5.3, and the definition of $\mathcal{J}_{-}(y)$ we obtain that $\mathcal{J}_{-}(y)=\mathcal{J}_{-}(x)$.

This fact shows that the CQ CRSC is preserved locally, as the set $\mathcal{J}_{-}(x)$ is constant in a neighborhood of a feasible point where CRSC holds. We are ready to show that CRSC implies an error bound.

TheOREM 5.5. If $x \in F$ verifies $C R S C$ and the functions $f_{i}, i=1, \ldots, m+p$, defining $F$ admit second derivatives in a neighborhood of $x$, then an error bound holds in a neighborhood of $x$.

Proof. First, let us recall that Lemma 5.3 states that the constraints in $\mathcal{J}_{-}(x)$ are actually equality constraints in a neighborhood of $x$. Hence, it is natural to consider the feasible set $F^{E}$ :

$$
F^{E}=\left\{y \in \mathbb{R}^{n} \mid f_{i}(y)=0 \forall i \in \mathcal{I} \cup \mathcal{J}_{-}(x), f_{j}(y) \leq 0 \forall j \in \mathcal{J}_{+}(x)\right\},
$$

which is equivalent to the original feasible set $F$ close to $x$. It is trivial to see that the CRSC point (with respect to $F$ ) $x$ verifies RCPLD as a feasible point of the set $F^{E}$. Now, using [5, Theorem 7], it follows that there exist $\alpha>0$ and a neighborhood $N(x)$ of $x$ such that for every $y \in N(x)$

$$
\min _{z \in F}\|z-y\|=\min _{z \in F^{E}}\|z-y\| \leq \alpha r^{E}(y)
$$

with

$$
r^{E}(y)=\max \left\{\left\|f_{\mathcal{I} \cup \mathcal{J}_{-}(x)}(y)\right\|_{\infty},\left\|\max \left\{0, f_{\mathcal{J}_{+}(x)}(y)\right\}\right\|_{\infty}\right\} .
$$

Now, from Lemma 5.2 we know that there are scalars $\lambda_{i}, i \in B$, and $\mu_{j}$, with $\mu_{j}>0$ for all $j \in \mathcal{J}_{-}(x)$, such that (5.1) holds. Let $\widehat{\mathcal{J}}$ be as in the proof of Lemma 5.4; that is, $\widehat{\mathcal{J}}$ is a subset of $\mathcal{J}_{-}(x)$ such that the set of gradients $\left\{\nabla f_{i}(x)\right\}_{i \in B \cup \widehat{\mathcal{J}}}$ is a basis for $\operatorname{span}\left\{\nabla f_{i}(x)\right\}_{i \in \mathcal{I} \cup \mathcal{J}_{-}(x)}$. Let us consider also the function

$$
h(y)=-\sum_{j \in \mathcal{J}_{-}(x) \backslash \widehat{\mathcal{J}}} \mu_{j} f_{j}(y) .
$$

Following the proof of Lemma 5.4, there are a neighborhood $N(x)$ of $x$, a neighborhood $Z$ of $\left(f_{B}(x), f_{\widehat{\mathcal{J}}}(x)\right)$, with $Z$ being convex, and a continuously differentiable function 
$g: Z \rightarrow \mathbb{R}$ such that (5.5)-(5.7) holds. By shrinking $N(x)$ if necessary, we can assume that the partial derivatives of $g$ will preserve the signs at $\left(f_{B}(x), f_{\widehat{\mathcal{J}}}(x)\right)$. That is, we may assume the existence of constants $0<\mu_{m} \leq \mu_{M}$ and $\lambda_{M}$ such that $\mu_{m} \leq \frac{\partial g}{\partial z_{j}}(z) \leq \mu_{M}$ for all $j \in \mathcal{J}_{-}(x)$ and $\left|\frac{\partial g}{\partial z_{i}}(z)\right| \leq \lambda_{M}$ for all $i \in B$ and all $z \in Z$.

Thus, from the convexity of $Z$ and the differentiability of $g$, we can apply the mean value theorem to see that, for each $y \in N(x)$, there exist $\xi_{y} \in Z$ between $(0,0)=\left(f_{B}(x), f_{\widehat{\mathcal{J}}}(x)\right)$ and $\left(f_{B}(y), f_{\widehat{\mathcal{J}}}(y)\right)$ such that

$$
g\left(f_{B}(y), f_{\widehat{\mathcal{J}}}(y)\right)=\sum_{i \in B \cup \widehat{\mathcal{J}}} \frac{\partial g}{\partial z_{i}}\left(\xi_{y}\right) f_{i}(y)
$$

This implies that

$$
-\sum_{j \in \mathcal{J}_{-}(x) \backslash \widehat{\mathcal{J}}} \mu_{j} f_{j}(y)=\sum_{i \in B \cup \widehat{\mathcal{J}}} \frac{\partial g}{\partial z_{i}}\left(\xi_{y}\right) f_{i}(y)
$$

and, for every $l \in \mathcal{J}_{-}(x) \backslash \widehat{\mathcal{J}}$, we can write

$$
-\mu_{l} f_{l}(y)=\sum_{i \in B \cup \widehat{\mathcal{J}}} \frac{\partial g}{\partial z_{i}}\left(\xi_{y}\right) f_{i}(y)+\sum_{j \in\left(\mathcal{J}_{-}(x) \backslash \widehat{\mathcal{J}}\right) \backslash\{l\}} \mu_{j} f_{j}(y) .
$$

Since $\mu_{l}>0$, it follows that

$$
\begin{aligned}
\left|f_{l}(y)\right| & \leq \frac{1}{\mu_{l}}\left(\sum_{i \in B \cup \widehat{\mathcal{J}}}\left|\frac{\partial g}{\partial z_{i}}\left(\xi_{y}\right)\right|\left|f_{i}(y)\right|+\sum_{j \in\left(\mathcal{J}_{-}(x) \backslash \widehat{\mathcal{J}} \backslash \backslash\{l\}\right.}\left|\mu_{j}\right| \max \left\{0, f_{j}(y)\right\}\right) \\
& \leq \frac{\max \left\{\mu_{M} ;\left|\mu_{j}\right|, j \in \mathcal{J}_{-}(x) \backslash \widehat{\mathcal{J}}\right\}}{\mu_{m}}\left(\sum_{i \in B \cup \widehat{\mathcal{J}}}\left|f_{i}(y)\right|+\sum_{j \in \mathcal{J}_{-}(x) \backslash \widehat{\mathcal{J}}} \max \left\{0, f_{j}(y)\right\}\right) .
\end{aligned}
$$

Thus, for all $l \in \mathcal{J}_{-}(x) \backslash \widehat{\mathcal{J}}$, there is a $K>0$ large enough such that

$$
\left|f_{l}(y)\right| \leq K \max \left\{\left|f_{i}(y)\right|, i \in \mathcal{I} ; \max \left\{0, f_{j}(y)\right\}, j \in \mathcal{J}\right\} .
$$

If $l \in \widehat{\mathcal{J}}$, from (5.12), we obtain a similar bound,

$$
\left|f_{l}(y)\right| \leq \widetilde{K} \max \left\{\left|f_{i}(y)\right|, i \in \mathcal{I} ; \max \left\{0, f_{j}(y)\right\}, j \in \mathcal{J}\right\},
$$

for some $\widetilde{K}>0$.

Using (5.13)-(5.14) and (5.10)-(5.11), we obtain the desired result.

5.2. Algorithmic applications of CPG. In this section, we show how the CPG condition can be used in the analysis of many algorithms for nonlinear optimization. The objective is to show that CPG can replace other more stringent CQs in the assumptions that ensure global convergence. We will show specific results for the main classes of algorithms for optimization, namely, sequential quadratic programming (SQP), interior point methods, augmented Lagrangians, and inexact restoration. 
5.2.1. Sequential quadratic programming. We start by extending the global convergence result of the general SQP method studied by Qi and Wei [38]. In their work, Qi and Wei introduced the CPLD CQ and extended convergence results for SQP methods that previously were based on the MFCQ. In order to do so, their main tool was the notion of AKKT sequences.

DeFINITION 5.6. We say that $\left\{x^{k}\right\}$ is an AKKT sequence of (NOP) if there is a sequence $\left\{\left(\lambda^{k}, \mu^{k}, \epsilon^{k}, \delta^{k}, \gamma_{k}\right)\right\} \in \mathbb{R}^{m} \times \mathbb{R}^{p} \times \mathbb{R}^{n} \times \mathbb{R}^{p} \times \mathbb{R}$ such that

$$
\left\{\begin{array}{l}
\nabla f_{0}\left(x^{k}\right)+\sum_{i=1}^{m} \lambda_{i} \nabla f_{i}\left(x^{k}\right)+\sum_{j=1}^{p} \mu_{j} \nabla f_{m+j}\left(x^{k}\right)=\epsilon^{k} \\
f_{m+j}\left(x^{k}\right) \leq \delta^{k}, j=1, \ldots, p \\
\mu^{k} \geq 0 \\
\mu_{j}^{k}\left(f_{m+j}\left(x^{k}\right)-\delta_{j}^{k}\right)=0, j=1, \ldots, p \\
\left|f_{i}\left(x^{k}\right)\right| \leq \gamma_{k}, \quad i=1, \ldots, m
\end{array}\right.
$$

where $\left\{\left(\epsilon^{k}, \delta^{k}, \gamma_{k}\right)\right\}$ converges to zero.

It is easy to see that AKKT sequences are closely related to AKKT feasible points from Definition 3.2. Actually, AKKT (feasible) points are exactly the limit points of AKKT sequences. Hence we can easily recast the results from [38] in terms of AKKT points.

In particular, Theorem 2.7 from [38], which ensures that limits of AKKT sequences are actually KKT, is just a particular case of Theorem 3.3 above, requiring CPLD, a more stringent CQ, in the place of CPG. Hence, we may use Theorem 3.3 to generalize some convergence results from [38], replacing CPLD by CPG.

In order to do so, let us recall the general SQP method from [38], as follows.

Algorithm 5.1 (general SQP). Let $C>0, x^{0} \in F, H_{0} \in \mathbb{R}^{n \times n}$ be a symmetric positive definite matrix.

1. (Initialization.) Set $k=0$.

2. (Computation of a search direction.) Compute $d^{k}$ solving the quadratic programming problem

$$
\begin{aligned}
\min & \frac{1}{2} d^{\prime} H_{k} d+\nabla f\left(x^{k}\right)^{\prime} d \\
\text { s.t. } & f_{i}\left(x^{k}\right)+\nabla f_{i}\left(x^{k}\right)^{\prime} d=0, i=1, \ldots, m, \\
& f_{i}\left(x^{k}\right)+\nabla f_{i}\left(x^{k}\right)^{\prime} d \leq 0, i=m+1, \ldots, m+p .
\end{aligned}
$$

If $d^{k}=0$, stop.

3. (Line search and additional correction.) Determine the step length $\alpha_{k} \in(0,1)$ and a correction direction $\bar{d}^{k}$ such that

$$
\left\|\bar{d}^{k}\right\| \leq C\left\|d^{k}\right\| .
$$

4. (Updates.) Compute a new symmetric positive definite Hessian approximation $H_{k+1}$. Set $x^{k+1}=x^{k}+\alpha_{k} d^{k}+\bar{d}^{k}$ and $k=k+1$. Go to step 1 .

As stated in [38], this algorithm is a general model for SQP methods, where specific choices for the Hessian approximations $H_{k}$, step length $\alpha_{k}$, and correction steps $\bar{d}^{k}$ are defined. Moreover, if the algorithm stops at step 2, then $x^{k}$ is a KKT point for (NOP). Hence, when analyzing such a method we need consider only the case where it generates an infinite sequence. The result below is a direct generalization of Theorem 4.2 in [38] where we use CPG instead of CPLD. 
THEOREM 5.7. Assume that the general SQP algorithm generates an infinite sequence $\left\{x^{k}\right\}$ and that this sequence has an accumulation point $x^{*}$. Let $\mathcal{L}$ be the index set associated with it, that is,

$$
\lim _{k \in \mathcal{L}} x^{k}=x^{*}
$$

Suppose that CPG holds at $x^{*}$ and that the Hessian estimates $H_{k}$ are bounded. If

$$
\liminf _{k \in \mathcal{L}}\left\|d_{k}\right\|=0
$$

then $x^{*}$ is a KKT point of (NOP).

Proof. We just follow the proof of Theorem 4.2 in [38] to see that it shows that under the assumptions above, $\left\{x^{k}\right\}_{k \in \mathcal{L}}$ is an AKKT sequence. Hence, as discussed before, $x^{*}$ is an AKKT point that is KKT whenever CPG holds by Theorem 3.3.

In order to present a concrete SQP algorithm that conforms to the assumptions of the theorem above, Qi and Wei recover the Panier-Tits SQP feasible algorithm for inequality constrained problems [37]. As pointed out by Qi and Wei, this method can be seen as a special case of the general SQP algorithm.

The Panier-Tits method depends on the validity of MFCQ on the computed iterates to be well defined. However, as pointed out by Qi and Wei, MFCQ does not need to hold at the limit points, where CPLD suffices. Once again we can generalize this result using CPG.

TheOrem 5.8. Consider the Panier-Tits feasible SQP method described in [38, Algorithm B]. Let $\left\{x^{k}\right\}$ be an infinite sequence generated by this method, and let $H_{k}$ be the respective Hessian approximations. Suppose that MFCQ holds at all feasible points that are not KKT and that the Hessian estimates $H_{k}$ are bounded, and let $x^{*}$ be an accumulation point of $\left\{x^{k}\right\}$, where CPG holds. Then $x^{*}$ is a KKT point of (NOP).

Proof. Once again we need only follow the proof from Theorem 5.3 in [38] and use Theorem 5.7 above instead of its particular case [38, Theorem 4.2].

Note that it is easy to build examples where MFCQ holds at all feasible points but one, where CPG holds and CPLD does not hold. See Figure 3.1 above. Hence the theorem above is a real generalization of Qi and Wei's result.

5.2.2. Interior point methods. Let us now turn our attention to how CPG can be used in the analysis of interior point methods for nonlinear optimization. In this context the usual CQ is the MFCQ $[10,12,14]$.

It is interesting to consider why the definition of CPLD did not result in the generalization of the convergence conditions for such methods. To this effect, let us focus on problems with inequality constraints only. In this case, it is natural to assume that the optimization problem satisfies a sufficient interior property, that is, that every local minimizer can be arbitrarily approximated by strictly feasible points. It is known from [16] that CPLD together with such a sufficient interior property is equivalent to MFCQ. Hence, it is fruitless to use CPLD to generalize results based on MFCQ in the context of interior point methods. Moreover, it is possible to replace CPLD with CRSC in the previous discussion since Lemma 5.3 shows that $\mathcal{J}_{-}(x)=\emptyset$ whenever CRSC and the sufficient interior property hold at a feasible point $x$; that is, MFCQ holds.

On the other hand, the example in Figure 3.2 shows that CPG and the sufficient interior property can hold together even when other CQs fail, in particular, MFCQ. 
Moreover, it was proved in [4] that the classic barrier method generates sequences with AKKT limit points. Hence, Theorem 3.3 shows that such limit points satisfy the KKT condition if CPG holds. This fact opens the path toward proving convergence of modern interior point methods under less restrictive CQs. In particular, we generalize below the convergence results for the quasi-feasible interior point method of Chen and Goldfarb [10].

This algorithm consists of applying a log-barrier strategy to solving the general optimization problem (NOP), yielding a sequence of subproblems $\left(\mathrm{FP}_{\zeta_{l}}\right)$, where the barrier sequence $\left\{\zeta_{l}\right\}$ should be driven to 0 :

$$
\begin{array}{ll}
\min & f_{0}(x)-\zeta_{l} \sum_{i=m+1}^{m+p} \log \left(-f_{i}(x)\right) \\
\text { s.t. } & f_{i}(x)=0, i=1, \ldots, m, \\
& f_{j}(x)<0, j=m+1, \ldots, m+p .
\end{array}
$$

Algorithm I in [10] uses an $\ell_{2}$-norm penalization to deal with the equality constraints in $\left(\mathrm{FP}_{\zeta_{l}}\right)$ and tries to solve it approximately employing a Newton-like approach. More formally, given a barrier parameter $\zeta_{l}>0$ and an error tolerance $\varepsilon_{l}>0$, Algorithm I tries to find $x^{l} \in \mathbb{R}^{n}, \lambda^{l} \in \mathbb{R}^{m}$, and $\mu^{l} \in \mathbb{R}^{p}$ such that $f_{j}\left(x^{l}\right)<0, j=m+1, \ldots, m+p$, and

$$
\begin{gathered}
\left\|\nabla f_{0}\left(x^{l}\right)+\sum_{i=1}^{m} \lambda_{i}^{l} \nabla f_{i}\left(x^{l}\right)+\sum_{j=1}^{p} \mu_{j}^{l} \nabla f_{m+j}\left(x^{l}\right)\right\| \leq \varepsilon_{l}, \\
\forall i=1, \ldots, m, \quad\left|f_{i}\left(x^{l}\right)\right| \leq \varepsilon_{l}, \\
\forall j=1, \ldots, p, \quad\left|f_{m+j}\left(x^{l}\right) \mu_{j}^{l}+\zeta_{l}\right| \leq \varepsilon_{l}, \\
\forall j=1, \ldots, p, \quad \mu_{j}^{l} \geq-\varepsilon_{l} .
\end{gathered}
$$

The conditions above are simply the termination criteria defining a successful run of Algorithm I as stated in [10, equation (3.13)]. Moreover, system (5.15)-(5.18) is an approximate version of the KKT conditions for $\left(\mathrm{FP}_{\zeta_{l}}\right)$.

Algorithm II is then defined in [10] as employing Algorithm I to approximately solve $\left(\mathrm{FP}_{\zeta_{l}}\right)$ for a sequence of barrier parameters $\zeta_{l}>0$ and error tolerance $\varepsilon_{l}>0$ both converging to 0 . We show below that it is possible to improve the convergence results of this method using CPG instead of MFCQ.

THEOREM 5.9. Assume that the standard assumptions A1-A2 of [10] hold; that $i s$, there exists a point $x_{0}$ such that $f_{i}\left(x_{0}\right)<0, i=m+1, \ldots, m+p$, and the functions $f_{i}, i=0, \ldots, m+p$, are twice continuously differentiable. Consider Algorithm II with sequences $\zeta_{l}>0$ and $\varepsilon_{l}>0$ both converging to zero. There are two possibilities:

1. For each $\zeta_{l}$ and $\varepsilon_{l}>0$, Algorithm I terminates satisfying conditions (5.15)(5.18), and in particular, Algorithm II generates a sequence $\left\{x^{l}\right\}$. If this sequence admits a limit point $x^{*}$, then it is feasible, and if $C P G$ with respect to (NOP) holds at $x^{*}$, it is also a KKT point of (NOP).

2. For some barrier parameter $\zeta_{l}$, the termination criteria of Algorithm I are never met. Let $\left\{x^{l, k}\right\}$ be the sequence computed by Algorithm I with penalty parameters associated with the equality constraints $\left\{r_{l, k}\right\}$. Suppose further that Assumptions A3-A4 of [10] hold; that is, the sequence $\left\{x^{l, k}\right\}$ and the modified Hessian sequence $\left\{\mathcal{H}^{l, k}\right\}$ used in Algorithm $\mathrm{I}$ is bounded. Let $x^{*}$ be 
a limit point of $\left\{x^{l, k}\right\}$. If $C P G$ with respect to the infeasibility problem

$$
\begin{array}{ll}
\min & \sum_{i=1}^{m} f_{i}(x)^{2} \\
\text { s.t. } & f_{i}(x) \leq 0, i=m+1, \ldots, m+p,
\end{array}
$$

holds at $x^{*}$ and $\left\{\nabla f_{i}\left(x^{*}\right)\right\}_{i=1}^{m}$ is linearly independent, then $x^{*}$ is a KKT point of such infeasibility problem.

Proof. First let us consider the case where Algorithm I successfully terminates conforming to (5.15)-(5.18) for all barrier parameters $\zeta_{l}$. Let $x^{*}$ be an accumulation point of $\left\{x^{l}\right\}$, and let $\mathcal{L}$ be the associated index set; that is, $x^{l} \rightarrow_{\mathcal{L}} x^{*}$.

To see that $x^{*}$ is feasible, we start noting that (5.16) and $\varepsilon_{l} \rightarrow 0$ ensure that $x^{*}$ respects all the equality constraints. Moreover, as a limit of points that obey (strictly) the inequalities, $x^{*}$ also conforms to the inequality constraints.

Now we show that $x^{*}$ is AKKT. Let us start with the observation that, for each $j=1, \ldots, p$, inequality (5.18) implies that either $\mu_{j}^{l} \rightarrow_{\mathcal{L}} 0$ or there is a $\delta_{j}>0$ and an infinite index set contained in $\mathcal{L}$, where $\mu_{j}^{l}>\delta_{j}$. Hence, repeating this procedure $p$ times, we can obtain a disjoint partition $I_{1} \cup I_{2}=\{1, \ldots, p\}$, an infinite index set $\mathcal{L}^{\prime} \subset \mathcal{L}$, and a $\delta>0$ such that for all $j \in I_{1}, \mu_{j}^{l} \rightarrow_{\mathcal{L}^{\prime}} 0$ and for all $j \in I_{2}, \mu_{j}^{l}>\delta$. In particular, if $j \notin \mathcal{A}\left(x^{*}\right)$, then inequality (5.17) together with $\zeta_{l} \rightarrow 0$ and $\varepsilon_{l} \rightarrow 0$ imply that $\mu_{j}^{l} \rightarrow_{\mathcal{L}} 0$. That is, $j \in I_{1}$.

Next we recover (5.15) and see that for $l \in \mathcal{L}^{\prime}$

$$
\left\|\nabla f_{0}\left(x^{l}\right)+\sum_{i=1}^{m} \lambda_{i}^{l} \nabla f_{i}\left(x^{l}\right)+\sum_{j \in I_{2}} \mu_{j}^{l} \nabla f_{m+j}\left(x^{l}\right)\right\| \leq \varepsilon_{\zeta_{l}}^{\prime},
$$

where $\varepsilon_{\zeta_{l}}^{\prime}$ is defined as $\varepsilon_{l}+\left\|\sum_{j \in I_{1}} \mu_{j}^{l} \nabla f_{m+j}\left(x^{l}\right)\right\|$. Using the continuity of the gradients of the constraints, $\varepsilon_{l} \rightarrow 0$, and for all $j \in I_{1}, \mu_{j}^{l} \rightarrow_{\mathcal{L}^{\prime}} 0$, it follows that $\varepsilon_{\zeta_{l}}^{\prime} \rightarrow_{\mathcal{L}^{\prime}} 0$ and therefore $x^{*}$ is AKKT.

Finally, we can use Theorem 3.3 to assert that the fact that the validity of CPG with respect to (NOP) holds at $x^{*}$ is enough to ensure that $x^{*}$ is a KKT point of (NOP).

Now consider the case where Algorithm I generates an infinite sequence for a fixed barrier parameter $\zeta_{l}$. There are two possibilities:

1. The penalty parameters $r_{l, k}$ are driven to infinity. In this case we follow the proof of [10, Theorem 3.6]. Dividing [10, equation (3.16)] by the previously defined $\alpha_{l, k}=\max \left\{r_{l, k},\left\|\mu_{l, k}\right\|_{\infty}\right\}$, where $\mu_{l, k}$ is the current multiplier estimate for the inequalities, it follows easily that $x^{*}$ is an AKKT point of the infeasibility problem above. Hence, it is a KKT point of such a problem if it fulfills CPG.

2. If the infinite sequence generated by Algorithm I is such that $r_{l, k}$ is bounded, then we follow the proof of [10, Lemma 3.8] to arrive at a contradiction with respect to the linear independence of the gradients of equality constraints.

Note that the assumption that CPG holds with linear independence of equality constraint gradients is a real weakening of MFCQ, as can be seen by the example in Figure 3.2 .

5.2.3. Augmented Lagrangians and inexact restoration. Finally, let us look at augmented Lagrangians algorithms. In particular, we consider the variant 
introduced in $[3,2]$ and show that it converges under CPG. This algorithm can solve problems in the form

(NOP-LA)

$$
\begin{array}{cl}
\min & f_{0}(x) \\
\text { s.t. } & f_{i}(x)=0, i=1, \ldots, m, \\
& f_{j}(x) \leq 0, j=m+1, \ldots, m+p, \\
& x \in X,
\end{array}
$$

where the set $X=\left\{x \mid \underline{f}_{i}(x)=0, i=1, \ldots, \underline{m}, \underline{f}_{j}(x) \leq 0, j=\underline{m}+1, \ldots, \underline{m}+\underline{p}\right\}$ is composed of easy constraints that can be enforced by a readily available solver.

In the original papers, the global convergence of the augmented Lagrangian algorithm was obtained assuming CPLD. Such results were recently extended to require only RCPLD [5]. In these works, the basic idea was to explore the fact that the algorithm can converge only to AKKT points and then use a special case of Theorem 3.3 above to show that the limit points are actually KKT points. The same line of reasoning can then be followed, requiring only CPG and generalizing the convergence result.

THEOREM 5.10. Let $x^{*}$ be a limit point of a sequence generated by the augmented Lagrangian algorithm described in $[3,2]$. Then one of the four conditions below holds:

1. $C P G$ with respect to the set $X$ does not hold at $x^{*}$.

2. $x^{*}$ is not feasible and it is a KKT point of the problem

$$
\begin{array}{ll}
\min & \sum_{i=1}^{m} f_{i}^{2}(x)+\sum_{j=m+1}^{m+p} \max \left\{0, f_{j}(x)\right\}^{2} \\
\text { s.t. } & x \in X .
\end{array}
$$

3. $x^{*}$ is feasible, but CPG fails at $x^{*}$ when taking into account the full set of constraints.

4. $x^{*}$ is $K K T$.

We close this section by mentioning that Theorem 3.3 also proves convergence of inexact restoration methods $[28,29,30,11]$ to KKT points under CPG, since limit points of sequences generated by these methods satisfy the LAGP optimality condition [4, 31], which implies AKKT [17].

6. Conclusion. We presented two new constraint qualifications that are weaker than the previous CQs based on constant rank and constant positive linear dependence.

The first CQ, which we called constant rank of the subspace component (CRSC), solves the open problem of identifying the specific set of gradients whose rank must be preserved locally and still ensure that the constraints are qualified. We achieved this by defining the set of active inequality constraints that resemble equalities, the set $\mathcal{J}_{-}$. We proved that under CRSC those inequalities are actually equalities locally and showed that an error bound holds.

The second CQ is more general and was called the constant positive generator $(\mathrm{CPG})$ condition. It basically asks that a generalization of the notion of a basis for a cone be preserved locally. This condition is very weak and can even hold in a point where Guignard's CQ fails in a neighborhood. Despite its weakness, we showed that this condition is enough to ensure that AKKT points conform to the KKT optimality conditions, and hence CPG can be used to extend global convergence results of many algorithms for nonlinear optimization. 
The definition of these two new CQs leads the way for several new research directions. For example, it would be interesting to investigate whether CRSC can be used to extend results on sensitivity and perturbation analysis that already exist for RCRCQ and CPLD [22, 23, 24, 33]. Another possibility would be to extend CRSC to the context of problems with complementarity or vanishing constraints [18, 21], as was done recently for CPLD in [19, 20]. Another interesting area of research is to search for alternative proofs or methods that allow us to drop the CQs that are stronger than CPG and that are still required in the convergence analysis of SQP and interior point methods presented in section 5.2.

Acknowledgment. The authors would like to thank the anonymous referees whose comments and suggestions have greatly improved the quality of this work.

\section{REFERENCES}

[1] J. Abadie, On the Kuhn-Tucker theorem, in Nonlinear Programming, John Wiley, New York, 1967, pp. 21-36.

[2] R. Andreani, E. G. Birgin, J. M. Martínez, and M. L. Schuverdt, On augmented Lagrangian methods with general lower-level constraints, SIAM J. Optim., 18 (2007), pp. $1286-1309$.

[3] R. Andreani, E. G. Birgin, J. M. Martínez, and M. L. Schuverdt, Augmented Lagrangian methods under the constant positive linear dependence constraint qualification, Math. Program., 111 (2008), pp. 5-32.

[4] R. Andreani, G. Haeser, and J. M. Martínez, On sequential optimality conditions for smooth constrained optimization, Optimization, 60 (2011), pp. 627-641.

[5] R. Andreani, G. Haeser, M. L. Schuverdt, and P. J. S. Silva, A relaxed constant positive linear dependence constraint qualification and applications, Math. Program., to appear.

[6] R. Andreani, J. M. Martínez, and M. L. Schuverdt, On the relation between constant positive linear dependence condition and quasinormality constraint qualification, J. Optim. Theory Appl., 125 (2005), pp. 473-483.

[7] M. S. BazaraA, H. D. Sherali, and C. M. Shetty, Nonlinear Programming: Theory and Algorithms, 3rd ed., John Wiley, Hoboken, NJ, 2006.

[8] D. P. Bertsekas, Nonlinear Programming, 2nd ed., Athena Scientific, Belmont, MA, 1999.

[9] D. P. Bertsekas, Convex Analysis and Optimization, Athena Scientific, Belmont, MA, 2003.

[10] L. Chen AND D. GoldFARB, Interior-point $\ell_{2}$-penalty methods for nonlinear programming with strong global convergence properties, Math. Program., 108 (2006), pp. 1-26.

[11] A. Fischer AND A. Friedlander, A new line search inexact restoration approach for nonlinear programming, Comput. Optim. Appl., 46 (2010), pp. 333-346.

[12] A. Forsgren, P. E. Gill, AND M. H. Wright, Interior methods for nonlinear optimization, SIAM Rev., 44 (2002), pp. 525-597.

[13] F. J. Gould And J. W. TOlLE, A necessary and sufficient qualification for constrained optimization, SIAM J. Appl. Math., 20 (1971), pp. 164-172.

[14] C. Grossmann, D. Klatte, and B. Kummer, Convergence of primal-dual solutions for the nonconvex log-barrier method without LICQ, Kybernetika, 20 (2004), pp. 571-584.

[15] M. Guignard, Generalized Kuhn-Tucker conditions for mathematical programming problems in a Banach space, SIAM J. Control, 7 (1969), pp. 232-241.

[16] G. HAESER, On the global convergence of interior-point nonlinear programming algorithms, Comput. Appl. Math., 29 (2010), pp. 125-138.

[17] G. Haeser And M. L. Schuverdt, On approximate KKT condition and its extension to continuous variational inequalities, J. Optim. Theory Appl., 149 (2011), pp. 125-138.

[18] T. Hoheisel AND C. KAnzow, First- and second-order optimality conditions for mathematical programs with vanishing constraints, Appl. Math., 52 (2007), pp. 495-514.

[19] T. Hoheisel, C. Kanzow, And A. Schwartz, Theoretical and numerical comparison of relaxation methods for mathematical programs with complementarity constraints, Math. Program., to appear.

[20] T. Hoheisel, C. Kanzow, And A. Schwartz, Mathematical programs with vanishing constraints: A new regularization approach with strong convergence properties, Optimization, 61 (2012), pp. 619-636 
[21] A. F. Izmailov And M. V. Solodov, Mathematical programs with vanishing constraints: Optimality conditions, sensitivity and a relaxation method, J. Optim. Theory Appl., 142 (2009), pp. 501-532.

[22] R. JANIN, Directional derivative of the marginal function in nonlinear programming, in Math. Program. Stud. 21, North-Holland, Amsterdam, The Netherlands, 1984, pp. 110-126.

[23] S. Lu, Implications of the constant rank constraint qualification, Math. Program., 126 (2009), pp. 365-392.

[24] S. Lu, Relation between the constant rank and the relaxed constant rank constraint qualifications, Optimization, 61 (2012), pp. 555-566.

[25] P. Malliavin, Géométrie différentielle intrinsèque, Hermann, Paris, 1972.

[26] O. L. Mangasarian, Nonlinear Programming, Classics Appl. Math. 10, SIAM, Philadelphia, 1994.

[27] O. L. Mangasarian and S. Fromovitz, The Fritz John necessary optimality conditions in the presence of equality and inequality constraints, J. Math. Anal. Appl., 17 (1967), pp. 37-47.

[28] J. M. MARTíNEZ, Inexact restoration method with Lagrangian tangent decrease and new merit function for nonlinear programming, J. Optim. Theory Appl., 111 (2001), pp. 39-58.

[29] J. M. Martínez And E. A. Pilotta, Inexact restoration algorithms for constrained optimization, J. Optim. Theory Appl., 104 (2000), pp. 135-163.

[30] J. M. Martínez AND E. A. Pilotta, Inexact restoration methods for nonlinear programming: Advances and perspectives, in Optimization and Control with Applications, L. Q. Qi, K. L. Teo, and X. Q. Yang, eds., Springer, New York, 2005, pp. 271-292.

[31] J. M. Martínez AND B. F. Svaiter, A practical optimality condition without constraint qualifications for nonlinear programming, J. Optim. Theory Appl., 118 (2003), pp. 117-133.

[32] L. Minchenko and S. Stakhovski, On relaxed constant rank regularity condition in mathematical programming, Optimization, 60 (2011), pp. 429-440.

[33] L. Minchenko and S. Stakhovski, Parametric nonlinear programming problems under the relaxed constant rank condition, SIAM J. Optim., 21 (2011), pp. 314-332.

[34] L. Minchenko and A. Tarakanov, On error bounds for quasinormal programs, J. Optim. Theory Appl., 148 (2011), pp. 571-579.

[35] J. Nocedal and S. J. Wright, Numerical Optimization, 2nd ed., Springer, New York, 2006.

[36] J.-S. PANG, Error bounds in mathematical programming, Math. Program., 79 (1997), pp. 299332.

[37] E. R. PAnier And A. L. Tits, On combining feasibility, descent and superlinear convergence in inequality constrained optimization, Math. Program., 59 (1993), pp. 261-276.

[38] L. QI AND Z. WEI, On the constant positive linear dependence condition and its application to SQP methods, SIAM J. Optim., 10 (2000), pp. 963-981.

[39] J. R. REAY, Unique minimal representations with positive bases, Amer. Math. Monthly, 73 (1966), pp. 253-261.

[40] R. T. RockAfEllar, Lagrange multipliers and optimality, SIAM Rev., 35 (1993), pp. 183-238.

[41] M. V. Solodov, Constraint qualifications, in Wiley Encyclopedia of Operations Research and Management Science, John Wiley, Hoboken, NJ, 2011. 